\title{
Chapter
}

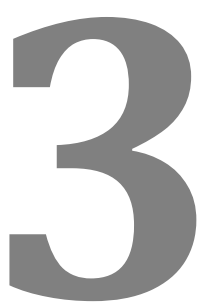

\section{THE IMPORTANCE OF AND DIFFERENT APPROACHES TO PERMEABILITY DETERMINATION}

\author{
Kin Y. Tam, Matěj Velický and Robert A.W. Dryfe
}

\section{Contents}

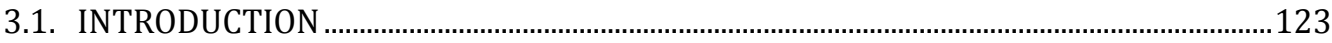

3.1.1. Permeability and the biopharmaceutical classification system ..........................123

3.1.2. The rationale for permeability determinations in drug discovery ....................126

3.2. THEORETICAL BACKGROUND OF PAMPA...............................................................................127

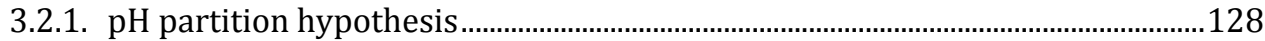

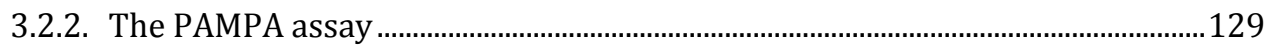

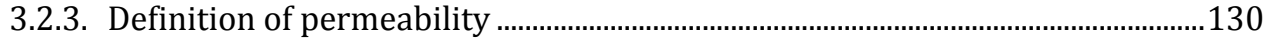

3.2.4. Advanced transport model for PAMPA.................................................................. 131

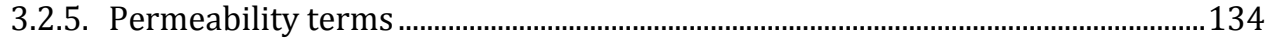

3.2.6. Permeability hydrodynamic model........................................................................ 134

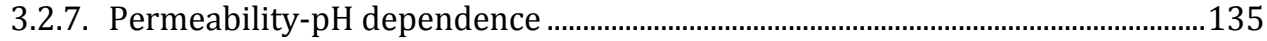

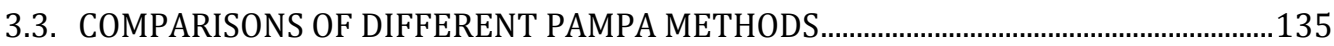




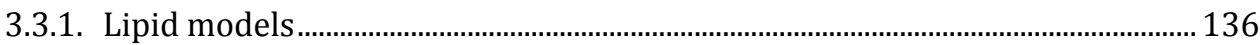

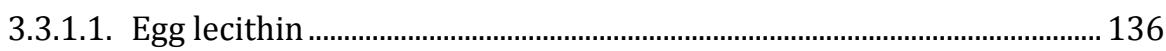

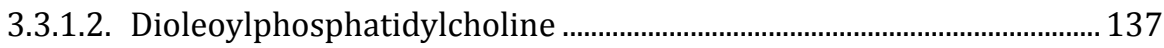

3.3.1.3. The double-sink method ............................................................................ 137

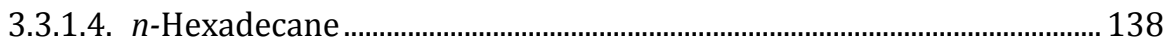

3.3.1.5. Mixture of lipids (biomimetic) ............................................................... 138

3.3.1.6. Lipid/oil/lipid tri-layer membrane........................................................ 138

3.3.1.7. Immobilised phospholipid vesicles ........................................................ 139

3.3.1.8. Lipid/cholesterol/octanol mixture.......................................................... 139

3.3.1.9. Three lipid-component model ............................................................... 140

3.3.1.10. Porcine brain tissue extract .................................................................. 140

3.3.2. Experimental procedures........................................................................... 140

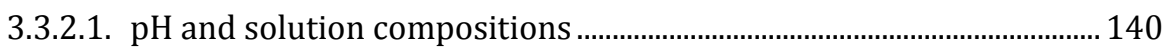

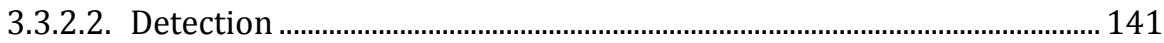

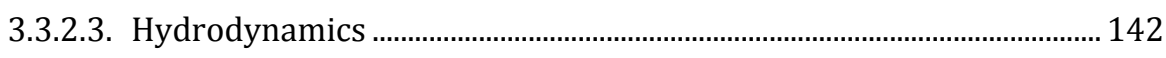

3.4. APPLICATIONS OF PAMPA DATA TO PREDICT AND/OR MODEL IN VIVO DATA .... 151

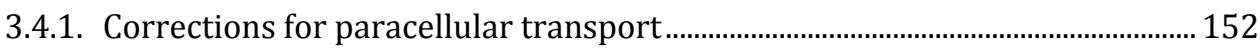

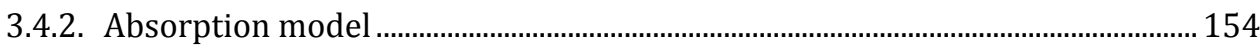

3.4.3. Correlation between in vitro permeability and the absorbed fraction of

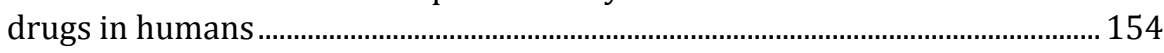

3.4.4. Prediction of the absorbed fraction of amphoteric drugs in humans............... 155

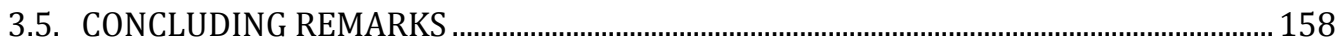

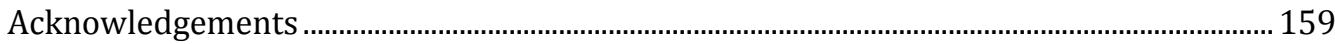

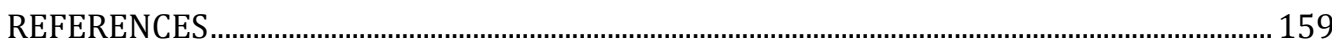




\subsection{INTRODUCTION}

The oral route remains the most preferable and popular way of administration for pharmaceuticals because of patient convenience. It offers the flexibility of using immediate or sustained release formulations. In developing new drugs, pharmaceutical companies generally focus their initial efforts on developing the drug as an oral candidate. However, if good oral absorption is difficult to achieve, other routes of administration, such as intravenous or intramuscular, may be developed as alternatives. Assuming metabolism and chemical degradation in the gastrointestinal (GI) tract are not significant, the rate and extent of absorption of a drug after oral administration depends on the concentration of the drug in the intestinal lumen and the permeability of the drug across the intestinal mucosa [1]. In many cases, especially when administered at a high dose, the drug concentration corresponds to the solubility of the drug in the lumen. Both solubility and permeability are dependent upon the structural characteristics of the drug, and are routinely determined in pharmaceutical research. The former is covered in Chapter 1 of this monograph. In this chapter, we will focus on the experimental aspects of permeability. First, we will briefly introduce drug permeability and absorption in the context of the biopharmaceutical classification system (BCS). Then, we will outline the rationale for permeability determination in the early phase of drug discovery. Particular emphasis will be placed on the different approaches based on artificial membranes for the determination of permeability.

\subsubsection{Permeability and the biopharmaceutical classification system}

Permeability and drug absorption are important factors governing the availability of a drug in the systematic circulation. Permeability refers to the flux of a drug across a membrane normalised with respect to the drug concentration at the membrane surface, while absorption generally refers to the movement of a drug into the bloodstream. The term absolute bioavailability offers a precise description of drug absorption, which could be defined as the fraction of an administered dose of drug that reaches the systemic circulation. When the dose is administered intravenously (iv), the bioavailability is $100 \%$. Figure 3.1 shows the typical plasma concentration time profiles obtained from an oral (po) or intravenous (iv) administration. For an orally administered dose (po), the absolute bioavailability $(F)$ can be defined as [2]: 


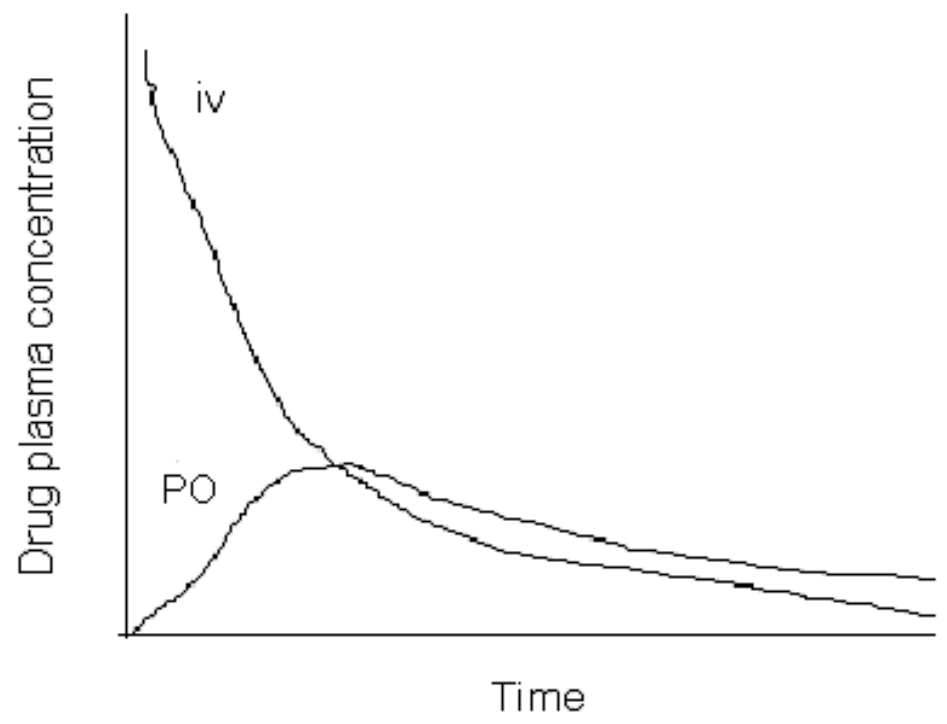

Figure 3.1. Plasma concentration as a function of time where the doses were administrated orally (po) or intravenously (iv)

$$
F=\frac{\overline{A U C}_{\mathrm{PO}}}{\overline{A U C}_{\mathrm{iv}}}
$$

where $\overline{A U C}$ represents the area under the curve normalised by the dose used by the respective route of administration. Taking hepatic first pass metabolism and gut wall metabolism into consideration, $F$ may be expressed as [2]:

$$
F=F_{\mathrm{a}} \times F_{\mathrm{h}} \times F_{\mathrm{g}}
$$

where $F_{\mathrm{a}}, F_{\mathrm{h}}$ and $F_{\mathrm{g}}$ represent, respectively, the fraction absorbed from the intestinal lumen, the fraction which escapes hepatic first pass metabolism and the fraction which escapes gut wall metabolism.

Thus, drug molecules invariably need to permeate through intestinal epithelial layers and various cellular barriers, then distribute to their sites of action and elicit a pharmacological response. Drug movement across membranes can be classified as transcellular, paracellular and active transport. Figure 3.2 shows a schematic of these routes. Transcellular transport is a passive process and is most likely to occur for lipophilic drugs. Paracellular transport is also passive in nature but is regarded as a size-restricted process for small molecules. Active transport generally invokes transporter proteins that actively expel or take up a certain class of molecules. The most common and widely studied transporter is the P-glycoprotein (P-gp) efflux transporter, which can limit the oral bioavailability or central nervous system (CNS) penetration of certain types of drugs. Other transporter proteins that facilitate the uptake of drug molecules have been reported, for example the organic anion transporters [3]. It has been suggested that 


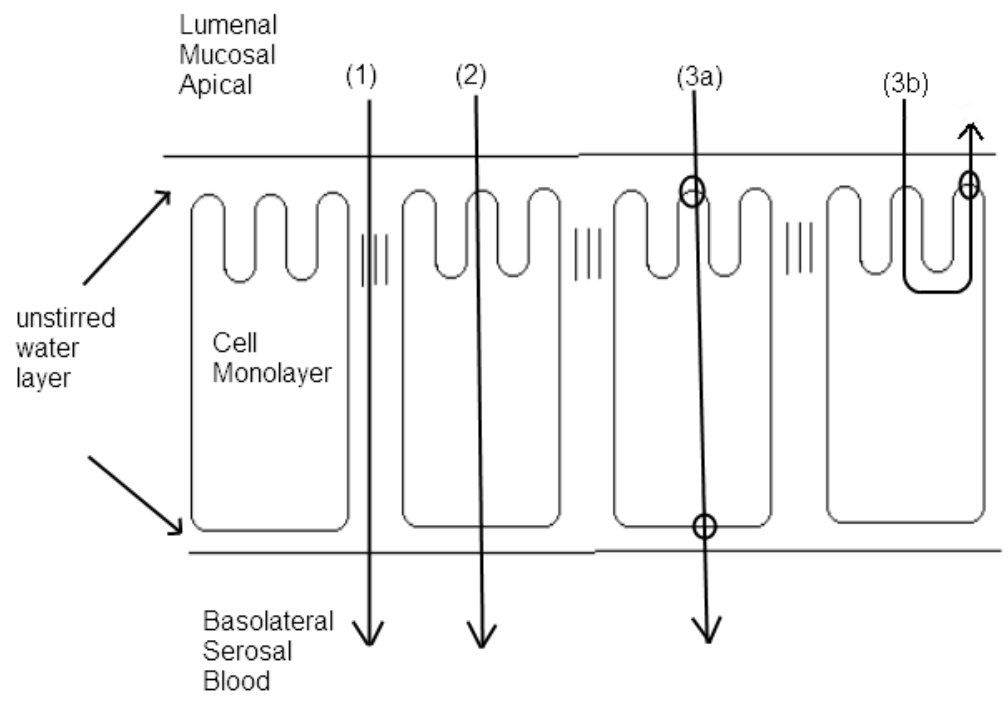

Figure 3.2. Routes of drug molecule transport across a membrane.

(1) Paracellular transport, (2) Transcellular transport, (3) Active transport:

(3a) carrier-mediated transport, (3b) efflux

carrier-mediated and active uptake of drugs might account for the majority of membrane transport processes in biological systems [3]. A recent compelling study revealed that both passive processes and facilitated/carrier-mediated processes coexist and contribute to drug transport across biological membranes [4].

The biopharmaceutical classification system is a classification scheme provided by the U.S. Food and Drug Administration (FDA) for correlating in vitro drug properties (permeability and solubility) with in vivo performance (bioavailability) [5,6]. As shown in Table 3.1, drugs can be categorised into four basic groups according to their solubility and permeability. This classification system can be used to assess the biopharmaceutical risks of investigational new drugs. For drug molecules with an immediate release solid oral dosage form and properties fitting in class I, it may be possible to waive the requirement for in vivo bioavailability and bioequivalence studies for any new formulations in the future, provided that the dissolution rate of the new dosage form remains rapid.

Table 3.1. The biopharmaceutical classification system (BCS)

\begin{tabular}{|l|l|}
\hline Class I & Class II \\
High Solubility & Low Solubility \\
High Permeability & High Permeability \\
\hline Class III & Class IV \\
High Solubility & Low Solubility \\
Low Permeability & Low Permeability \\
\hline
\end{tabular}


In the assessment of drug permeability class, the absolute bioavailability in humans is the most obvious parameter to consider. For instance, high bioavailability (i.e. > $90 \%$ ) generally implies high permeability. However, when high bioavailability cannot be achieved, the FDA recommends various experimental approaches to determine the permeability class. These include: (1) in vivo intestinal perfusion studies in humans; (2) in vivo or in situ intestinal perfusion studies using suitable animal models; (3) in vitro permeation studies using excised human or animal intestinal tissues; or (4) in vitro permeation studies across a cultured monolayer of animal (e.g. MDCK cells) or human epithelial cells (e.g. Caco-2 cells). It has been noted that in vivo or in situ animal models and in vitro methods using cultured monolayers of epithelial cells should be limited to the study of passively transported drugs. This is typically reflected by a linear (pharmacokinetic) relationship between the dose and measures of bioavailability, or the lack of concentration dependency on in vitro permeability across monolayers epithelial cells which are known to express efflux transporters (e.g. P-gp). The determination of permeability class is accomplished by a rankorder relationship using a sufficient number of model drugs tested using the same experimental approach. For example, a test drug may be regarded as highly permeable when its permeability is equal to or greater than that of a selected model drug with high permeability.

\subsubsection{The rationale for permeability determinations in drug discovery}

The BCS provides a rigorous framework to rationalise the interplay between the permeability/solubility and the in vivo performance (bioavailability) of a tested drug. As highlighted in Section 3.1.1, the recommended experimental approaches for determining permeability require the use of animal/human subjects or tissues. This would be viable for investigational new drugs in clinical development, as the project normally deals with a single active pharmaceutical ingredient. However, in the early stage of a discovery project, the primary focus is to develop and optimise a lead chemical series against a particular drug target of interest. Structural diversity is regarded as an important criterion to enable a thorough exploitation of the structure-activity relationship and pharmaceutical properties. Consequently, the direct and routine use of these experimental approaches in early stage drug discovery projects may exceed the available resources in some discovery organisations.

In 1998, Kansy et al. introduced the parallel artificial membrane permeability assay (PAMPA) [7]. Since then, the technique has received considerable attention in the pharmaceutical industry. PAMPA offers a simple physicochemical measure of passive permeability for research compounds. The throughput is higher than that of other in vitro cell-based permeability assays (e.g. Caco-2 or MDCK cell lines) and it is relatively cheap to run [8-10]. PAMPA is a useful tool in early phase discovery projects where permeability is seen to be an issue in the chemical series of interest. For instance, in combination with other in vitro assays, such as metabolic assessments, aqueous solubility and plasma protein 
binding, PAMPA can form a part of the testing cascade to evaluate the suitability of research compounds.

As the lead chemical series progresses towards the lead optimisation phase, it is of great interest to identify the most promising lead compounds to evaluate their pharmacokinetic and pharmacodynamic properties in pre-clinical animal models. Poor oral bioavailability could arise because of a number of reasons, including metabolic liability (hepatic metabolism), efflux transport or other active processes, gut wall metabolism, as well as suboptimal physical properties such as poor solubility or poor permeability. PAMPA data helped to assess the permeability of the test research compounds in the absence of other detrimental factors such as metabolism and efflux. Other aforementioned in vitro assays could be carried out in parallel as part of the testing cascade. This holistic approach allows the discovery project to spot issues quickly, triggers the design and synthesis of the next round analogues to moderate any identified issues and helps with the prioritisation of the most promising lead compounds for pharmacokinetic studies, with the goal of reducing the use of live animals on those compounds that are unlikely to show a reasonable level of oral exposure.

In the next two sections, we will turn our attention to the theoretical aspect of PAMPA, followed by a brief discussion on different PAMPA models developed since 1998. Finally, in Section 3.4, we will highlight two examples on the use of PAMPA data in pharmaceutical research.

\subsection{THEORETICAL BACKGROUND OF PAMPA}

For an orally administered dose, the absorption of the drug predominately occurs in the small intestine. The $\mathrm{pH}$ of the GI tract ranges from about 1 in the stomach to 6.5 in the small intestine, whereas bloodstream $\mathrm{pH}$ is about 7.4. According to the pH partition hypothesis (see Section 3.2.1), it is thought that only neutral species transfer across the lipophilic intestinal epithelial layers. Generally speaking, a neutral drug tends to be less soluble in a water-based environment than an ionised drug of a similar size. To circumvent this problem, an ionisable centre can be introduced. On the other hand, a large fraction of ionised species results in higher solubility in the aqueous phase (GI tract or bloodstream), but decreases the rate of drug transfer across the intestinal epithelial layers. In the case of a monoprotic drug, the fraction of neutral species, $f_{\mathrm{N}}$, can be calculated using the Henderson-Hasselbalch equation:

$$
f_{\mathrm{N}}=\frac{1}{\left(1+10^{ \pm\left(\mathrm{pH}-\mathrm{p} K_{\mathrm{a}}\right)}\right)}
$$

where the sign in the exponent is + for acids and - for bases. $\mathrm{p} K_{\mathrm{a}}$ represents the acid dissociation constant. Table 3.2 shows the equations for calculating the neutral fraction and ionised fraction for monoprotic and diprotic drugs. 
Table 3.2. Equations for calculating the neutral and ionised fractions for ionisable drugs

\begin{tabular}{|c|c|c|}
\hline Charge type & $\begin{array}{c}\text { Acid } \\
\text { dissociation } \\
\text { constant }\end{array}$ & $\begin{array}{l}\text { Equations for calculating the neutral }\left(f_{\mathrm{N}}\right)^{\mathrm{a}} \text { and } \\
\text { ionised }\left(f_{\mathrm{i}}(+) \text {, cation; } f_{\mathrm{i}}(-) \text {, anion }\right)^{\mathrm{b}} \text { fractions }\end{array}$ \\
\hline Monoprotic acid & $\mathrm{p} K_{\mathrm{a}}$ & $\begin{array}{c}f_{\mathrm{N}}=\frac{1}{\left(1+10^{\left(\mathrm{pH}-\mathrm{pK} K_{\mathrm{a}}\right)}\right)} \\
f_{\mathrm{I}}(-)=1-f_{\mathrm{N}}\end{array}$ \\
\hline Monoprotic base & $\mathrm{p} K_{\mathrm{a}}$ & $\begin{array}{c}f_{\mathrm{N}}=\frac{1}{\left(1+10^{-\left(\mathrm{pH}-\mathrm{p} K_{\mathrm{a}}\right)}\right)} \\
f_{\mathrm{I}}(-)=1-f_{\mathrm{N}}\end{array}$ \\
\hline Diprotic acid & $\begin{array}{c}\mathrm{p} K_{\mathrm{a}, 1}, \mathrm{p} K_{\mathrm{a}, 2} \\
\left(\mathrm{p} K_{\mathrm{a}, 2}>\mathrm{p} K_{\mathrm{a}, 1}\right)\end{array}$ & $\begin{array}{c}f_{\mathrm{N}}=\frac{1}{\left(1+10^{\left(\mathrm{pH}-\mathrm{p} K_{\mathrm{a}, 1}\right)}+10^{\left(2 \mathrm{pH}-\mathrm{p} K_{\mathrm{a}, 1}-\mathrm{p} K_{\mathrm{a}, 2}\right)}\right)} \\
f_{\mathrm{I}}(-)=1-f_{\mathrm{N}}\end{array}$ \\
\hline Diprotic base & $\begin{array}{c}\mathrm{p} K_{\mathrm{a}, 1}, \mathrm{p} K_{\mathrm{a}, 2} \\
\left(\mathrm{p} K_{\mathrm{a}, 2}>\mathrm{p} K_{\mathrm{a}, 1}\right)\end{array}$ & $\begin{array}{c}f_{\mathrm{N}}=\frac{1}{\left(1+10^{-\left(\mathrm{pH}-\mathrm{p} K_{\mathrm{a}, 2}\right)}+10^{-\left(2 \mathrm{pH}-\mathrm{p} K_{\mathrm{a}, 1}-\mathrm{p} K_{\mathrm{a}, 2}\right)}\right)} \\
f_{\mathrm{I}}(+)=1-f_{\mathrm{N}}\end{array}$ \\
\hline $\begin{array}{c}\text { Diprotic } \\
\text { amphotyle/zwitterion }\end{array}$ & $\begin{array}{c}\mathrm{p} K_{\mathrm{a}, 1}, \mathrm{p} K_{\mathrm{a}, 2} \\
\left(\mathrm{p} K_{\mathrm{a}, 2}>\mathrm{p} K_{\mathrm{a}, 1}\right)\end{array}$ & $\begin{array}{c}f_{\mathrm{N}}=\frac{1}{\left(1+10^{-\left(\mathrm{pH}-\mathrm{p} K_{\mathrm{a}, 1}\right)}+10^{\left(\mathrm{pH}-\mathrm{p} K_{\mathrm{a}, 2}\right)}\right)} \\
f_{\mathrm{I}}(+)=\frac{1}{\left(1+10^{\left(\mathrm{pH}-\mathrm{p} K_{\mathrm{a}, 1}\right)}+10^{\left(2 \mathrm{pH}-\mathrm{p} K_{\mathrm{a}, 1}-\mathrm{p} K_{\mathrm{a}, 2}\right)}\right)} \\
f_{\mathrm{I}}(-)=\frac{1}{\left(1+10^{-\left(\mathrm{pH}-\mathrm{p} K_{\mathrm{a}, 2}\right)}+10^{-\left(2 \mathrm{pH}-\mathrm{p} K_{\mathrm{a}, 1}-\mathrm{p} K_{\mathrm{a}, 2}\right)}\right.}\end{array}$ \\
\hline
\end{tabular}

a This represents the sum of the fraction of the neutral and zwitterionic species for a diprotic zwitterion

b These represent the sum of the fraction of single and double charged species for a diprotic acid or base

\subsection{1. $\mathrm{pH}$ partition hypothesis}

The commonly acknowledged $\mathrm{pH}$ partition hypothesis described by Jacobs in 1940 assumes that only the neutral fraction of a weak electrolyte can cross the cell membrane [11]. This hypothesis was extended to transport across biological membranes by Shore et al., who published work on the gastric secretion of drugs [12]. This report studied the appearance of drug molecules in the gastric juices of dogs after they had been administered intravenously. As the drug concentration changed with its dissociation constant, it was deduced that only the non-ionised 
fraction of the drug molecule could be absorbed through cell membranes. This assumption was applied to drug transport across the intestinal epithelium by the same research group [13] and was also tested in alternative systems such as diffusion through polymeric membranes [14]. The $\mathrm{pH}$ partition hypothesis is a good rule of thumb for the permeation/partitioning of drugs. It has, however, been criticised for being an oversimplification and for neglecting the fact that ionised species also permeate biological membranes although at a much slower rate than non-ionised species [15]. A number of reports in the 1990s showed evidence of partitioning of the ionised form of drug molecules to phosphatidylcholine bilayers [16-18]. In addition, paracellular transport through tight junctions in the intestinal epithelia was shown to be more common for cationic than other forms of drug molecules [19]. Similar observations were made for the epithelial monolayer in the Caco-2 assay, where significant transport of the ionised fraction was found when the neutral fraction concentration dropped to less than $10 \%$ [20].

\subsubsection{The PAMPA assay}

The PAMPA method is based on passive diffusion of a drug molecule between two aqueous compartments, the donor and the acceptor, separated by an artificial membrane. The assay was originally designed as a model to mimic transcellular transport of orally administered drugs across the intestinal epithelium. The schematic of the assay concept is shown in Figure 3.3. The membrane is soaked with a lipid solution in a non-polar organic solvent. The drug is present in the donor compartment at an appropriate concentration (usually tens to hundreds of $\mu \mathrm{M})$. The PAMPA is typically carried out in a commercial 96-well microtitre plate (acceptor) and a filter plate where the filter is coated with a lipid solution (donor). This enables the study of a large set of compounds in one experimental batch. A drug molecule, originally present in the aqueous donor compartment, diffuses across the hydrophobic membrane into the aqueous acceptor compartment. The drug concentrations in both compartments are then detected by an appropriate analytical technique and permeability is determined.

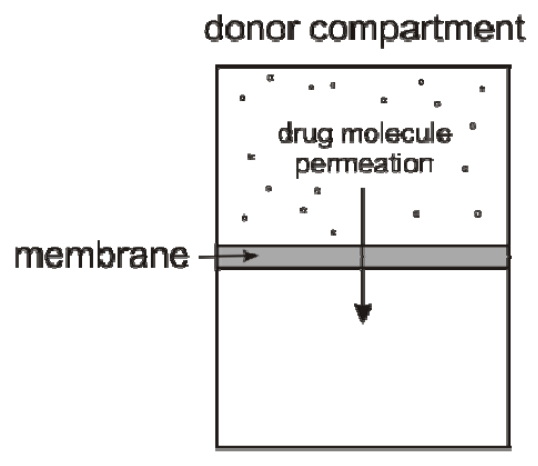

acceptor compartment

Figure 3.3. Schematic diagram of the PAMPA method 
Different PAMPA models have been developed over the last decade. These will be discussed in Section 3.3. In the next section (Section 3.2.3), we will direct our attention to the theoretical aspect of permeability.

\subsubsection{Definition of permeability}

Permeability is regarded as a rate parameter which can be broadly defined as the flux of material (normalised against the concentration) transported across a defined surface. This may be exemplified using in vivo intestinal perfusion studies in humans. In this approach, a local anaesthetic is applied to an awake human subject, and a multichannel tube is introduced into the intestine [21]. With the multichannel tube, two small balloons are inflated, $10 \mathrm{~cm}$ apart, to create a closed segment, which is rinsed and then perfused with a marker compound and the test drug. The human jejunal effective permeability, $P_{\text {eff, }}$ is inferred from the loss of the test drug from the perfusate, which can be described as below:

$$
P_{\text {eff }}=\frac{Q_{\text {in }}\left(C_{\text {in }}-C_{\text {out }}\right)}{C_{\text {out }} \times 2 \pi r l}
$$

where $Q_{\text {in }}$ represents the volume flow rate of the perfusate entering the segment. $C_{\text {in }}$ and $C_{\text {out }}$ represent, respectively, the concentrations of the test drug entering and leaving the segment. The symbols $r$ and $l$ represent, respectively, the radius and length of the segment. Note that the units of permeability are expressed as $\mathrm{cm} / \mathrm{s}$.

In cases of in vitro permeation studies using excised intestinal tissues or monolayers of cultured human epithelial cells (e.g. Caco-2), the experimental setup is conceptually the same as PAMPA, as shown in Figure 3.3. Calculations of the effective permeability, $P_{\mathrm{e}}$, may be made according to the following equation [22]:

$$
P_{\mathrm{e}}=\frac{\mathrm{d} M}{\mathrm{~d} t} \frac{1}{A \times C_{\mathrm{D}}(0)}
$$

where $\mathrm{d} M / \mathrm{d} t$ represents the steady-state appearance rate of the test drug in the acceptor compartment (in units of mol/s). $A$ is the exposed membrane area (in units of $\left.\mathrm{cm}^{2}\right)$ and $C_{\mathrm{D}}(0)$ is the initial drug concentration in the donor compartment (in units of mol/ $\mathrm{cm}^{3}$ ). Again, the units of permeability are in $\mathrm{cm} / \mathrm{s}$.

Equations 3.4 and 3.5 assume the unidirectional transport of the test drug from the intestinal lumen to the bloodstream, and from the donor to the acceptor, respectively. Mass transport in the opposite direction is ignored. These are generally regarded as one-way flux equations, and are valid under sink conditions. In the PAMPA method, sink conditions are not always maintained and drug retention in the membrane may introduce errors in the measurement. Avdeef has summarised various transport models with different limitations regarding reverse flux and membrane retention [23]. In particular, the commonly used two-way flux equation in PAMPA methods could be used to described the 
acceptor compartment concentration $\left(C_{\mathrm{A}}(\mathrm{t})\right)$ as a function of time $(t)$, which is written as follows [24]:

$$
C_{\mathrm{A}}(t)=\frac{M-M_{\mathrm{mem}}}{V_{\mathrm{D}}+V_{\mathrm{A}}}+\left(C_{\mathrm{A}}(0)-\frac{M-M_{\mathrm{mem}}}{V_{\mathrm{D}}+V_{\mathrm{A}}}\right) \exp \left(-P_{e} A\left(\frac{1}{V_{\mathrm{D}}}+\frac{1}{V_{\mathrm{A}}}\right) t\right)
$$

where $M$ and $M_{\text {mem }}$ represent, respectively, the total quantity of the drug in the system (in mol), and the sample loss to the membrane. The symbols $V_{\mathrm{D}}$ and $V_{\mathrm{A}}$ represent, respectively, the donor and acceptor volumes.

\subsubsection{Advanced transport model for PAMPA}

In this section, we present an advanced transport model for the PAMPA method [25]. In addition to reverse flux and membrane retention which were dealt with by the two-way flux equation, this enhanced model takes into account the initial lag-time, $\tau_{\mathrm{LAG}}$, (steady-state establishment across the donor-membrane-acceptor system). Correction for the lag-time is important as neglecting this factor leads to an underestimation of the permeability of lipophilic molecules (and an overestimation of the permeability of hydrophilic molecules). This will be discussed in Section 3.3.2.3.

The distribution of the drug in the donor-membrane-acceptor system is depicted in Figure 3.4 for (a) an infinitely stirred and (b) a well-stirred system. In this example, we assume the distribution coefficient of the drug compound to the
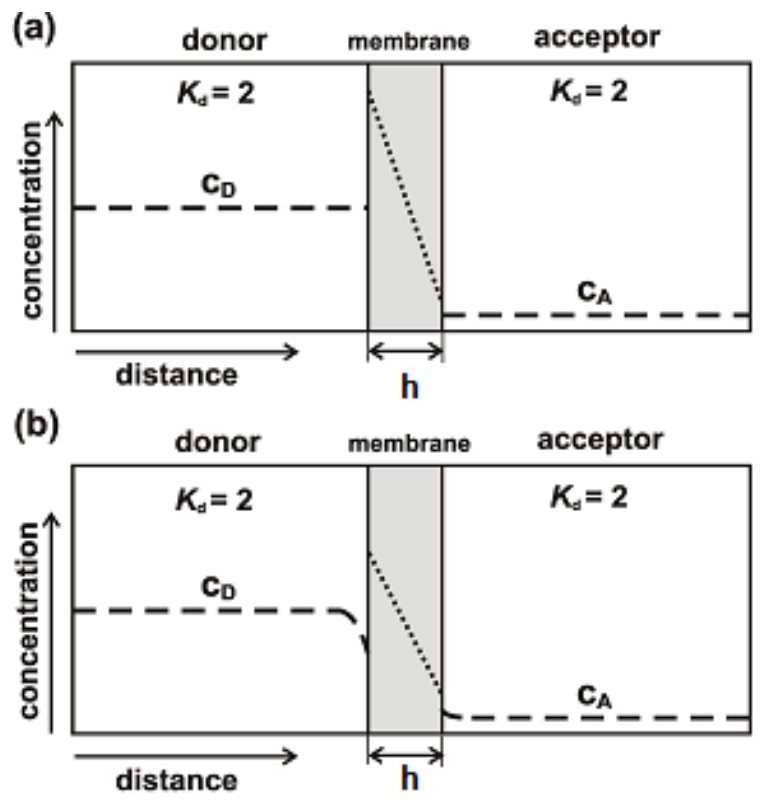

Figure 3.4. Schematic diagram of the concentration profile across a donormembrane-acceptor tri-layer for the case of: (a) zero UWL thickness,

(b) non-zero UWL thickness [25]. 
membrane phase $\left(K_{d}\right)$ to be 2 . For the infinitely stirred case (Figure $3.4 \mathrm{a}$ ), the unstirred water layer (UWL) is virtually non-existent, while for the well-stirred case, the concentration profiles are bent in close proximity to the membraneliquid interfaces due to the presence of the UWL. Both cases assume time $\geq \tau_{\mathrm{LAG}}$ and therefore a linear concentration distribution within the membrane. Fick's first law is considered valid in such cases.

The following relationships were used in the model derivation:

(i) Fick's first law applied to a homogeneous membrane:

$$
J(t)=P_{\mathrm{e}}^{\mathrm{D} \rightarrow \mathrm{A}} C_{\mathrm{D}}(t)-P_{\mathrm{e}}^{\mathrm{A} \rightarrow \mathrm{D}} C_{\mathrm{A}}(t)
$$

where $J(t)$ is the time-dependent flux of the solute across the membrane and $P_{\mathrm{e}}^{\mathrm{D} \rightarrow \mathrm{A}}$ and $P_{\mathrm{e}}^{\mathrm{A} \rightarrow \mathrm{D}}$ denote the effective permeability coefficients for the donor-toacceptor and acceptor-to-donor transport, respectively. Note that these permeability terms may differ if there is a $\mathrm{pH}$ gradient between the donor and acceptor phases. Finally, $C_{\mathrm{D}}(t)$ and $\mathrm{C}_{\mathrm{A}}(t)$ are the time dependent bulk concentrations of the solute in the donor and acceptor compartments, respectively.

(ii) Diffusive flux at the membrane/donor interface:

$$
J(t)=\frac{-V_{\mathrm{D}}}{A} \frac{\mathrm{d} C_{\mathrm{D}}(t)}{\mathrm{d} t}
$$

(iii) Mass balance:

$$
V_{\mathrm{D}} C_{\mathrm{D}}(0)=V_{\mathrm{D}} C_{\mathrm{D}}(t)+V_{\mathrm{A}} C_{\mathrm{A}}(t)+V_{\mathrm{m}} C_{\mathrm{m}}(t)
$$

where $V_{\mathrm{D}}, V_{\mathrm{A}}$ and $V_{\mathrm{m}}$ are the volumes of the donor, acceptor and membrane, respectively, $A$ is the membrane area, $C_{D}(0)$ is the initial bulk solute concentration in the donor compartment and $C_{\mathrm{m}}(t)$ is the time-averaged solute concentration within the membrane.

(iv) Finally, the expression for fractional membrane retention $R_{f}$ is defined as:

$$
R_{\mathrm{f}}=1-\frac{V_{\mathrm{D}} C_{\mathrm{D}}(t)+V_{\mathrm{A}} C_{\mathrm{A}}(t)}{V_{\mathrm{D}} C_{\mathrm{D}}(0)}
$$

By combining Equations 3.9 and 3.10, we obtain the mass balance equation containing the fractional membrane retention $R_{\mathrm{f}}$ :

$$
V_{\mathrm{D}} C_{\mathrm{D}}(0)=V_{\mathrm{D}} C_{\mathrm{D}}(t)+V_{\mathrm{A}} C_{\mathrm{A}}(t)+R_{\mathrm{f}} V_{\mathrm{D}} C_{\mathrm{D}}(0)
$$

In the case of the infinitely stirred system with zero UWL thickness, the effective permeability coefficients can be expressed as follows:

$$
P_{\mathrm{e}}^{\mathrm{D} \rightarrow \mathrm{A}}=P_{\mathrm{m}}^{\mathrm{D} \rightarrow \mathrm{A}}=\frac{D_{\mathrm{m}} K_{\mathrm{d}}^{\mathrm{D}}}{h}
$$




$$
P_{\mathrm{e}}^{\mathrm{A} \rightarrow \mathrm{D}}=P_{\mathrm{m}}^{\mathrm{A} \rightarrow \mathrm{D}}=\frac{D_{\mathrm{m}} K_{\mathrm{d}}^{\mathrm{A}}}{h}
$$

where $D_{\mathrm{m}}$ is the diffusion coefficient of the solute within the membrane and $P_{\mathrm{m}}^{\mathrm{D} \rightarrow \mathrm{A}}$ and $P_{\mathrm{m}}^{\mathrm{A} \rightarrow \mathrm{D}}$ are the membrane permeability coefficients of the solute for donor-to-acceptor and acceptor-to-donor flux, respectively. $K_{\mathrm{d}}^{\mathrm{D}}$ and $K_{\mathrm{d}}^{\mathrm{A}}$ are the solute distribution coefficients between the membrane-donor and membraneacceptor, respectively, and $h$ is the membrane thickness. Combining Equations 3.7, 3.8, 3.11, 3.12 and 3.13, we obtain a linear ordinary differential equation:

$$
\frac{\mathrm{d} C_{\mathrm{D}}(t)}{\mathrm{d} t}+a C_{\mathrm{D}}(t)-b=0
$$

where:

$$
a=\frac{A P_{\mathrm{e}}^{\mathrm{D} \rightarrow \mathrm{A}}}{V_{\mathrm{D}}}\left(1+\frac{K_{\mathrm{d}}^{\mathrm{A}}}{K_{\mathrm{d}}^{\mathrm{D}}} \frac{V_{\mathrm{D}}}{V_{\mathrm{A}}}\right), \quad b=\frac{A P_{\mathrm{e}}^{\mathrm{D} \rightarrow \mathrm{A}}}{V_{\mathrm{A}}} \frac{K_{\mathrm{d}}^{\mathrm{A}}}{K_{\mathrm{d}}^{\mathrm{D}}} C_{\mathrm{D}}(0)\left(1-R_{\mathrm{f}}\right)
$$

The differential equation, Equation 3.14, can be solved to obtain the analytical solution for times $\geq \tau_{\mathrm{LAG}}$ :

$$
\ln k=-a\left(t-\tau_{\mathrm{LAG}}\right)
$$

where $k$ is a function of the measured solute concentration in the acceptor compartment:

$$
k=\frac{C_{\mathrm{D}}(0)\left(1-R_{\mathrm{f}}\right)-\frac{b}{a}-\frac{V_{\mathrm{A}}}{V_{\mathrm{D}}} C_{\mathrm{A}}(t)}{C_{\mathrm{D}}\left(\tau_{\mathrm{LAG}}\right)-\frac{b}{a}}
$$

where $C_{\mathrm{D}}\left(\tau_{\mathrm{LAG}}\right)$ is the solute bulk concentration in the donor compartment at time $t=\tau_{\mathrm{LAG}}$.

Assuming (1) $K_{\mathrm{d}}^{\mathrm{D}} \approx K_{\mathrm{d}}^{\mathrm{A}}$ and (2) $\tau_{\mathrm{LAG}} \approx 0$, Equation 3.17 could be simplified to the two-way flux equation (Equation 3.6). When the $\mathrm{pH}$ values of the donor and the acceptor compartments differ, $K_{\mathrm{d}}^{\mathrm{D}}$ and $K_{\mathrm{d}}^{\mathrm{A}}$ are not the same for ionisable molecules. In such cases, they could be determined from independent shake-flask partition experiments [26]. The lag-time correction will be discussed in Section 3.3.2.3, together with experimental data. In a mathematical sense, $\tau_{\mathrm{LAG}}$ could be regarded as a constant of integration, so it could be treated as any time in the course of the experiment where the above derivations hold. In practice, $\tau_{\mathrm{LAG}}$ should be chosen as the time at which the function of Equation 3.17 starts to exhibit linear behaviour. From the slope of the ln $k$ vs. time plot (i.e. the constant $a$ defined in Equation 3.15), one directly obtains the effective permeability value, as in the case of the two-way flux equation (Equation 3.6). 


\subsubsection{Permeability terms}

The scenario shown in Figure 3.4a can only be regarded as an ideal situation where stirring is carried out at infinite speed, and the UWL thickness reduces to virtually zero. The solute concentrations in the bulk donor (acceptor) solution and at the donor-membrane (acceptor-membrane) interface are therefore the same. In reality, there is always a contribution of the UWL to the permeation, and the bulk solute concentration in the donor compartment is always higher than at the donor-membrane interface, as depicted in Figure 3.4b (likewise, the bulk solute concentration in the acceptor is always lower than the acceptormembrane interface concentration). Therefore, what one measures is the effective permeability coefficient consisting of two independent terms: membrane permeability, $P_{m}$, corresponding purely to transport through the membrane (no UWL contribution), and unstirred water layer permeability, $P_{\mathrm{u}}$, corresponding to transport through the two unstirred water layers adjacent to the membrane. The effective permeability can be described as the inverse of the total resistance to passive transport across the tri-layer UWL(donor)-membraneUWL(acceptor) system and is broken down into the following two terms [23,27]:

$$
\frac{1}{P_{\mathrm{e}}}=\frac{1}{P_{\mathrm{u}}}+\frac{1}{P_{\mathrm{m}}}
$$

where $P_{\mathrm{u}}$ is the combined permeability of both the donor and acceptor UWLs and $P_{\mathrm{m}}$ is the membrane permeability. In analogy to electrical circuits, the inverse permeability terms can be regarded as resistances, which are additive in series.

\subsubsection{Permeability hydrodynamic model}

Approximate relationships between the UWL permeability and stirring rate have been used in previous Caco- 2 and PAMPA studies $[27,28]$ :

$$
P_{\mathrm{u}}=K_{\mathrm{P}} \omega^{\alpha}
$$

where $\omega$ is the angular velocity of stirring, $\alpha$ is the hydrodynamic exponent and $K_{\mathrm{P}}$ is a constant composed of the aqueous diffusion coefficient of the solute $D_{\text {aq }}$, the aqueous kinematic viscosity $v$ and the geometrical factors of the permeation cell. The explicit form for the case of transport to the surface of a rotating-disc was found by Levich [29]. The unstirred water layer thickness, $\delta_{u}$, can be expressed as follows:

$$
\delta_{\mathrm{u}}=1.61 D_{\mathrm{aq}}{ }^{1 / 3} v^{1 / 6} \omega^{-1 / 2}
$$

The Levich solution applied to the single UWL permeability yields:

$$
P_{u}=0.62 D_{\mathrm{aq}}^{2 / 3} v^{-1 / 6} \omega^{\alpha}
$$

In pharmaceutical applications, $\alpha$ is often treated as an empirical value, ranging from 0.7 to 1.0 , and is usually determined by the best fit in UWL permeability 
analysis [27-28,30]. Combining Equations 3.21 and 3.22, which yields $\alpha=0.5$, we have a relationship between the unstirred water layer thickness and unstirred water layer permeability:

$$
P_{\mathrm{u}}=\frac{D_{\mathrm{aq}}}{\delta_{\mathrm{u}}}
$$

Equation 3.23 is an analogue to Equations 3.12 and 3.13 where the membrane permeability, membrane diffusion coefficient and the membrane thickness are substituted for the UWL permeability, aqueous diffusion coefficient and the UWL thickness, respectively. Combining Equations 3.19 and 3.22 , the $P_{\mathrm{e}}-\omega$ dependency can be written as follows [31-34]:

$$
\frac{1}{P_{\mathrm{e}}}=\frac{1}{0.62 D_{\mathrm{aq}}{ }^{2 / 3} v^{-1 / 6} \omega^{\alpha}}+\frac{1}{P_{\mathrm{m}}}
$$

Measuring $P_{\mathrm{e}}$ at two or more different stirring rates allows extrapolation to the infinite angular velocity, where $P_{\mathrm{u}}=0$ and $P_{\mathrm{e}}=P_{\mathrm{m}}$, which yields the membrane permeability value.

\subsubsection{Permeability-pH dependence}

For ionisable compounds, the membrane permeability $\left(P_{\mathrm{m}}\right)$ depends on $\mathrm{pH}$. Equation 3.25 defines the $\mathrm{pH}$ independent intrinsic permeability $P_{0}$, i.e. the permeability of the neutral species, assuming that the $\mathrm{pH}$ partition hypothesis [12] is valid:

$$
P_{m}=P_{0} f_{N}
$$

where $f_{\mathrm{N}}$ is the fraction of the neutral species, which is defined in Table 3.2. Combining Equations 3.19 and 3.25, we can write the following relationship:

$$
\frac{1}{P_{\mathrm{e}}}=\frac{1}{P_{\mathrm{u}}}+\frac{1}{P_{0} f_{N}}
$$

Note that $f_{\mathrm{N}}$ is a function of $\mathrm{pH}$ and the $\mathrm{p} K_{\mathrm{a}}(\mathrm{s})$ of the drug. If two or more $P_{e}$ values are measured at different $\mathrm{pH}$ values (for example within $\pm 2 \mathrm{pH}$ units from the $\mathrm{p} K_{\mathrm{a}}$ ), one should be able to deduce the $P_{0}$, and $P_{\mathrm{u}}$ values using Eq- $3.26[27,35]$.

\subsection{COMPARISONS OF DIFFERENT PAMPA METHODS}

Since the introduction of PAMPA by Kansy et al. [7], the technique has evolved considerably. These advancements can be loosely classified as follows:

- Lipid models.

- Experimental procedures, including the control of hydrodynamics and solution compositions.

- Applications of PAMPA data to predict and/or model in vivo data in pharmaceutical research. 
In most cases, the goal for improving/modifying lipid models and/or experimental procedures is to enhance the predictive/modelling power of PAMPA data. Hence, these three categories are somehow inter-related. In this section, we seek to focus on methodologies (i.e. lipid models and experimental procedures). In the next section, we will then turn our attention to selected examples of applying PAMPA data in pharmaceutical research.

\subsubsection{Lipid models}

Different research groups have developed lipid models for use in PAMPA. These are summarised in Table 3.3, and described in the following subsections.

Table 3.3. Summary of the PAMPA lipid models described in this chapter

\begin{tabular}{|c|c|c|c|c|}
\hline Lipid model & Composition $^{a}$ & Filterb & Solvent & Agitation \\
\hline Egg lecithin [7] & $10 \%$ Egg lecithin & PVDF & $n$-Dodecane & None \\
\hline $\begin{array}{l}\text { Dioleoylphosphat- } \\
\text { idylcholine [24] }\end{array}$ & $2 \% \mathrm{DOPC}$ & PVDF & $n$-Dodecane & Shaking \\
\hline $\begin{array}{c}\text { Double-sink method } \\
{[23,36-37]}\end{array}$ & $\begin{array}{l}20 \% \text { Phospholipid } \\
\text { mixture }\end{array}$ & PVDF & $n$-Dodecane & $\begin{array}{c}\text { Individual } \\
\text { well } \\
\text { stirring }\end{array}$ \\
\hline n-Hexadecane [35] & $100 \% n$-Hexadecane & Polycarbonate & $n$-Dexadecane & Shaking \\
\hline $\begin{array}{l}\text { Mixture of lipids } \\
\text { (biomimetic) [40] }\end{array}$ & $\begin{array}{c}0.8 \% \text { PC, } 0.8 \% \text { PE, } \\
0.2 \% \text { PS, } 0.2 \% \text { PI, } 1 \% \\
\text { CHO }\end{array}$ & PVDF & 1,7-Octadiene & None \\
\hline $\begin{array}{l}\text { Lipid/oil/lipid tri-layer } \\
\text { membrane [43] }\end{array}$ & Neat DOPC & PVDF & $\begin{array}{l}\text { Small amount } \\
\text { of hexadecane }\end{array}$ & None \\
\hline $\begin{array}{l}\text { Immobilised } \\
\text { phospholipid } \\
\text { vesicles [45] }\end{array}$ & $\begin{array}{l}\text { Neat phospholipid } \\
\text { vesicles }\end{array}$ & $\begin{array}{l}\text { Cellulose ester } \\
\text { filters }\end{array}$ & None & Shaking \\
\hline $\begin{array}{l}\text { Lipid/cholesterol/octa- } \\
\text { nol mixture [46] }\end{array}$ & $\begin{array}{c}1.7 \% \text { Egg PC, } 2.1 \% \text { CHO, } \\
96.2 \% \text { octanol }\end{array}$ & $\begin{array}{c}\text { cellulose } \\
\text { nitrate-acetate } \\
\text { mixture filter }\end{array}$ & Octanol & Flow \\
\hline $\begin{array}{l}\text { Three lipid-component } \\
\text { model [48] }\end{array}$ & $\begin{array}{l}2.6 \% \text { PS18:1, } 0.9 \% \\
\text { PC18:1, 1.5 \% CHO }\end{array}$ & PVDF & $n$-Dodecane & None \\
\hline $\begin{array}{c}\text { Porcine brain tissue } \\
\text { extract [49] }\end{array}$ & $2 \%$ Porcine brain extract & PVDF & $n$-Dodecane & None \\
\hline $\begin{array}{l}\text { DOPC - Dioleoylphospha } \\
\text { PS - phosphatidylserine } \\
\text { glycero-3-[phospho- } L \text {-se } \\
\text { PVDF - Poly(vinylidene }\end{array}$ & $\begin{array}{l}\text { atidylcholine, PC - phospha } \\
\text { PI - phosphatidylinositol, } \\
\text { erine], PC18:1 - 1,2-dioleoy } \\
\text { fluoride) }\end{array}$ & $\begin{array}{l}\text { ylcholine, } P \\
0 \text { - cholest } \\
\text { n-glycero-3- }\end{array}$ & $\begin{array}{l}\text { Dhosphatidyl } \\
\text { PS18:1 - 1,2 } \\
\text { sphocholine }\end{array}$ & $\begin{array}{l}\text { anolamine } \\
\text { oleoylsn- } \\
\text { c18:1) }\end{array}$ \\
\hline
\end{tabular}

\subsubsection{Egg lecithin}

This model refers to the lipid system reported in the PAMPA paper by Kansy et al. [7]. The artificial membrane was a hydrophobic filter (PVDF filter from Millipore; pore size $0.22-0.45 \mu \mathrm{m}$ ) impregnated with a $10 \%$ solution of egg lecithin in $n$-dodecane. The selection of egg lecithin was to mimic the phospholipid composition of mammalian membranes. Permeation experiments were performed at $\mathrm{pH} 6.5$ and 7.4 (same pH values for both donor and acceptor) on a selected number of 
marketed drugs, over 15 hours without stirring. The measured PAMPA flux was shown to correlate with the percent human absorption values via a hyperbolic function.

Kansy's PAMPA model forms the basis for subsequent developments. Most of these new models vary in terms of the compositions of the lipid solution or filter materials with the aim to enhance PAMPA data, and/or to mimic the transport processes other than that of the intestinal epithelial cell membrane (e.g. bloodbrain barrier).

\subsubsection{Dioleoylphosphatidylcholine}

This was the first commercially available PAMPA lipid model developed by Avdeef's group [24], based on a $2 \%$ solution of a synthetic phospholipid, dioleoylphosphatidylcholine (DOPC), dissolved in $n$-dodecane. Membrane retention was considered via the two-way flux equation. Solution agitation during the permeation studies was accomplished by orbital shaking. In addition, LC-MS and UV detection methods were compared, and the resultant permeability data were shown to be highly correlated. Development by the same group has subsequently led to an improved formulation, the double-sink method, which is described in the next section (Section 3.3.1.3).

\subsubsection{The double-sink method}

The double-sink PAMPA method (DS-PAMPA) has subsequently superseded the $2 \%$ DOPC model [23,36-37]. In particular, Avdeef has demonstrated that the PAMPA data obtained using the DS-PAMPA model correlates well with several absorption-related parameters [36,38-39]. Selected examples will be highlighted in Section 3.4 .

DS-PAMPA uses a pH gradient between the donor and acceptor solution (usually 5.0-7.4 and 7.4, respectively), with the addition of chemical scavengers to the acceptor to mimic the presence of serum proteins in blood. Anionic surfactant, such as sodium lauryl sulfate (SLS), at a concentration of $35 \mathrm{mM}$ (c.a. the saturated micelle concentration at room temperature) is used as chemical scavenger [23]. The effect of SLS is generally more pronounced in basic and neutral compounds, while the permeation of acidic compounds do not change significantly [23], suggesting electrostatistic interactions between the anionic surfactants and the permeating compounds may have a role in the transport mechanism. This results in double-sink conditions that suppress reverse-flux permeation from the acceptor to the donor compartment and shorten the experimental time. The lipid solution consists of a $20 \%$ phospholipid mixture in $n$-dodecane. A further advantage of this PAMPA model is the introduction of individual well stirring, which enables the unstirred water layer (UWL) to be controlled on an empirical basis [27]. With this model, the assay time can be shortened considerably by lowering the UWL thickness (and therefore resistance against diffusion). This is particularly useful for lipophilic molecules, where the membrane permeability $\left(P_{\mathrm{m}}\right)$ is comparable to the unstirred water layer permeability $\left(P_{\mathrm{u}}\right)$. 


\subsubsection{4. $\quad n$-Hexadecane}

Faller et al. introduced a lipid model based on hexadecane (HDM-PAMPA, in the absence of any phospholipids), impregnated on a thin $(10 \mu \mathrm{m})$ polycarbonate filter of low porosity, in order to better resemble the thickness of bilayer membranes found in living cells [35]. However, the absence of any phospholipids implies that the membrane could be much less permeable than the membrane used in DS-PAMPA and other models (see below). Similar to the case of the DOPC model (Section 3.3.1.2), the sandwich plate is constantly shaken during the permeation assay. Permeability-pH profiles have been determined for a set of model drugs. It has been shown that HDM permeability (the maximum value within the $\mathrm{pH}$ range of $\mathrm{pH} 4$-8) provides the best correlation with the absorbed fraction in humans. The correction for the effects of the unstirred water layer was discussed and analysed in light of the permeability-pH dependence (Equation 3.26).

\subsubsection{Mixture of lipids (biomimetic)}

Sugano et al. used a mixture of various lipids dissolved in 1,7-octadiene to mimic the content of mammalian cell walls in a so-called "bio-mimetic" PAMPA (BMPAMPA] [40]. Specifically, the lipid solution consists of $0.8 \%$ phosphatidylcholine (PC), $0.8 \%$ phosphatidylethanolamine (PE), 0.2\% phosphatidylserine (PS), $0.2 \%$ phosphatidylinositol (PI) and $1.0 \%$ cholesterol (CHO). It has been shown that the permeability data obtained at $\mathrm{pH} 5.5$ and/or 6.5 (but not at pH 7.4) provided a good prediction of the dose fraction absorbed in humans for a set of marketed drugs. The permeation study was carried out without agitation. In a follow-up study [41], a larger set of drug molecules was investigated using the BM-PAMPA model. The measured permeability data were modified using the Renkin function [42] to account for paracellular transport. The modified permeability data were found to give a good prediction of passive intestinal absorption [41].

\subsubsection{Lipid/oil/lipid tri-layer membrane}

Chen et al. reported a new procedure to prepare artificial membranes that contain a reduced amount of organic solvent [43]. It was hypothesised that the excess amount of organic solvents used in other lipid models might lead to compound retention within the membrane. The artificial membrane is formed on PVDF by three coating steps. First, $1 \mu \mathrm{L}$ of $n$-hexadecane is dispersed into PVDF, with hexane as a carrier to form the middle oil layer. DOPC, pre-dissolved in hexane, is then coated on either side of the membrane. Due to the volatile nature of hexane, it evaporates immediately after coating, thus leaving the lipid layers exposed on the surface. A set of drug molecules has been tested using this new lipid model, and the results appeared to outperform two other lipid models (the $2 \%$ DOPC model and DS-PAMPA) in terms of predicting/correlating the percent absorbed in humans and Caco-2 permeability. It has been noted that molecules such as antipyrine, caffeine, ketoprofen, metoprolol and naproxen are not under- 
predicted by this model, while they are under-predicted by the other two lipid models. These molecules are relatively small ( $\mathrm{MW}<270 \mathrm{~g} \mathrm{~mol}^{-1}$ ), so it would be interesting to see if this good correlation holds for a larger set of drug-like molecules.

The lipid/oil/lipid tri-layer is commercially available as a pre-coated PAMPA plate (marketed by BD Biosciences). Avdeef et al. have recently reported an evaluation of this pre-coated PAMPA plate [44]. It is interesting to note that the high permeability of antipyrine is reproducible across a range of $\mathrm{pH}$ values. However, this could be due to the fragile thin membrane, which contains leaky water pores. Avdeef et al. have estimated the void volume in the PVDF filter to be about $2.6 \mu \mathrm{L} /$ well [44]. It is plausible that the $1 \mu \mathrm{L}$ hexadecane volume used in the pre-coated plates may not be enough to fully plug the inner volume of the filter. As a result, some water channels may form under gradient-pH conditions, leading to higher measured permeability values for relatively small polar molecules.

\subsubsection{Immobilised phospholipid vesicles}

Flaten et al. have developed a novel procedure to directly deposit a tight barrier of liposomes on a filter support without the use of an inert solvent such as hexadecane [45]. The preparation of the membrane involves a multi-step extrusion-centrifugation procedure. Egg phosphatidylcholine was used for preparing the liposomes. The permeation study was carried out from 5 hours up to 12 hours (with shaking to control the UWL thickness). A set of drug molecules has been evaluated in this model, alongside other lipid models, such as DSPAMPA and BM-PAMPA. Membrane retention was found to be surprisingly low in this model, presumably due to the absence of any organic solvent in the membrane. It has been shown that the permeability data obtained from the liposome model correlate well with the percent absorbed in humans. Moreover, the performance of this model appears to be better than the other two PAMPA models, which may offer a useful model for further evaluation. However, the complex nature of the membrane preparation procedure may limit its routine use in other laboratories.

\subsubsection{Lipid/cholesterol/octanol mixture}

Corti et al. evaluated different lipid mixtures and filter membranes to develop the optimal lipid model [46]. Permeation experiments were carried out in a flowthrough diffusion cell, using a reference model compound, naproxen. It has been shown that, with a lipid mixture containing $2.1 \%$ cholesterol, $1.7 \%$ egg phosphatidylcholine and $96.2 \%$ octanol impregnated in a cellulose nitrateacetate mixture filter, the measured PAMPA permeability matches exactly with the Caco-2 permeability. The lipid model has been applied to a set of structurally diverse drugs [47]. The measured permeability values were found to be correlated well with the percent absorbed in humans. 


\subsubsection{Three lipid-component model}

Polli et al. have recently reported the evaluation of a three lipid-component model [48]. In brief, the lipid solution consists of $2.6 \%$ 1,2-dioleoylsn-glycero-3[phospho-L-serine] (PS18:1), $0.9 \%$ 1,2-dioleoylsn-glycero-3-phosphocholine (PC18:1) and $1.5 \%$ cholesterol in $n$-dodecane. Five microlitres of this solution were impregnated into a PVDF filter to prepare the artificial membrane. As PS18:1 is an anionic lipid, the model is referred to as A-PAMPA, where the letter A denotes anionic. A-PAMPA was designed to mimic the lipid composition of the enterocyte plasma membrane. This lipid model has been applied to a set of drugs and the permeability values were compared with the corresponding Caco-2 permeability values. It has been shown that the A-PAMPA model has correctly classified highly permeable drugs, while the classification has not always been correct for drugs of low permeability.

\subsubsection{Porcine brain tissue extract}

Di et al. applied $2 \%$ porcine brain tissue extract dissolved in $n$-dodecane as their model lipid [49]. The system was tested using a set of commercial drugs and research compounds. The permeation study was carried out without agitation. It was shown that the model was able to differentiate CNS+ (high brain penetration) from CNS- (low brain penetration) compounds with a high success rate.

Avdeef and Tsinman undertook a study to compare the permeability data obtained from the DOPC-, HDM- and DS-PAMPA models [50]. Forty drug molecules were considered in their investigation. It was shown that PAMPA permeability values consistently ranked in magnitude according to: DS > DOPC > HDM. This has been rationalised by the fact that the higher lipid content in DSPAMPA may help the permeation process by offering a source of hydrogen bonding with the membrane phase. In combo models based on Abraham descriptors ( $\alpha$ - solute H-bond acidity; $\beta$ - solute H-bond basicity) were developed to correlate the intrinsic permeability values $\left(P_{0}\right)$ between the HDM and DS models, as well as the DOPC and DS models. As far as we are aware, no systematic comparative study on different lipid models has been reported in the open literature. It would be interesting to extend the comparison as carried out by Avdeef and Tsinman [50] to other recently developed lipid models using a representative set of drug molecules.

\subsubsection{Experimental procedures}

\subsubsection{1. $\mathrm{pH}$ and solution compositions}

PAMPA studies are normally carried out in aqueous buffer systems. Both the donor and acceptor compartments contain aqueous buffer at selected $\mathrm{pH}$ values (Figure 3.3). For instance, the $\mathrm{pH}$ of the donor and acceptor could be adjusted to 6.5 and 7.4 to mimic the physicochemical conditions in the small intestine and bloodstream, respectively. Some investigators prefer to determine the $\mathrm{pH}$ - 
permeability profiles [35] by changing the donor and acceptor $\mathrm{pH}$ values to mimic the $\mathrm{pH}$ changes along the small intestine. This approach provides more information on permeability changes in different $\mathrm{pH}$ environments, which would be useful for mechanistic studies on the permeation process. In cases where the solubility of the drug molecules is low, it would be possible to introduce a small percentage of co-solvent into the assay buffer to avoid compound precipitation during the assay [40]. However, care must be taken in terms of the affects of the co-solvent on the permeability values. It is sensible to repeat the study with several different co-solvent compositions to rationalise the effects on the permeability values.

As mentioned in Section 3.3.1.3, the double-sink method introduces a chemical scavenger to the acceptor, which is another variant in solution composition. This suppresses the back-flux permeation from the acceptor to the donor compartment and shortens the experimental time.

\subsubsection{Detection}

The methods of detection of the permeating compound vary from ultraviolet (UV) spectrophotometry to more selective liquid chromatography/mass spectrometry (LC/MS) methods [24]. One of the reasons for the selection of LC/MS is mainly the large number of "difficult" molecules, which are unsuitable for UV analysis because of poor sensitivity. The high-throughput screening PAMPA method allows ex situ analysis of the donor and acceptor plates. At a particular time point, the donor-acceptor sandwich is disassembled and quantified to determine the concentration of the permeating compound. To generate multiple time point data, the PAMPA experiment can start with identical, multiple copies of donor-acceptor plate sandwiches, each of which could be disassembled and quantified at selected time points.

Recently, a rotating permeation cell was developed to investigate the physicochemical aspects of drug permeation [25]. It employs in situ UV measurements of the solute combined with stirring of both the acceptor and donor compartments in a system with defined symmetric geometry. The acceptor $\mathrm{pH}$ is kept at 7.4; the donor $\mathrm{pH}$ is varied between 3.0 and 10.0. The standard permeation time is as short as 20-30 min, and stirring rates in the range of 200-1500 rpm are used (see Figure 3.5 for the experimental schematic). The chosen membrane model comprised of $1.5 \%$ dioleoylphosphatidylcholine and $0.5 \%$ stearic acid dissolved in 1,9-decadiene and impregnated on a hydrophobic poly(vinylidene fluoride) (PVDF) filter. Despite the fact that the cell only consists of a single permeation channel, the in situ UV measurement and the well-controlled stirring in both the donor and acceptor compartments enable the device to be used for the study of drug permeation under controlled hydrodynamic conditions and to investigate the time-dependent transport of the compound observed in situ. The in situ time dependency of the permeability data as determined using this rotating permeation cell will be discussed in the next section (Section 3.3.2.3). 


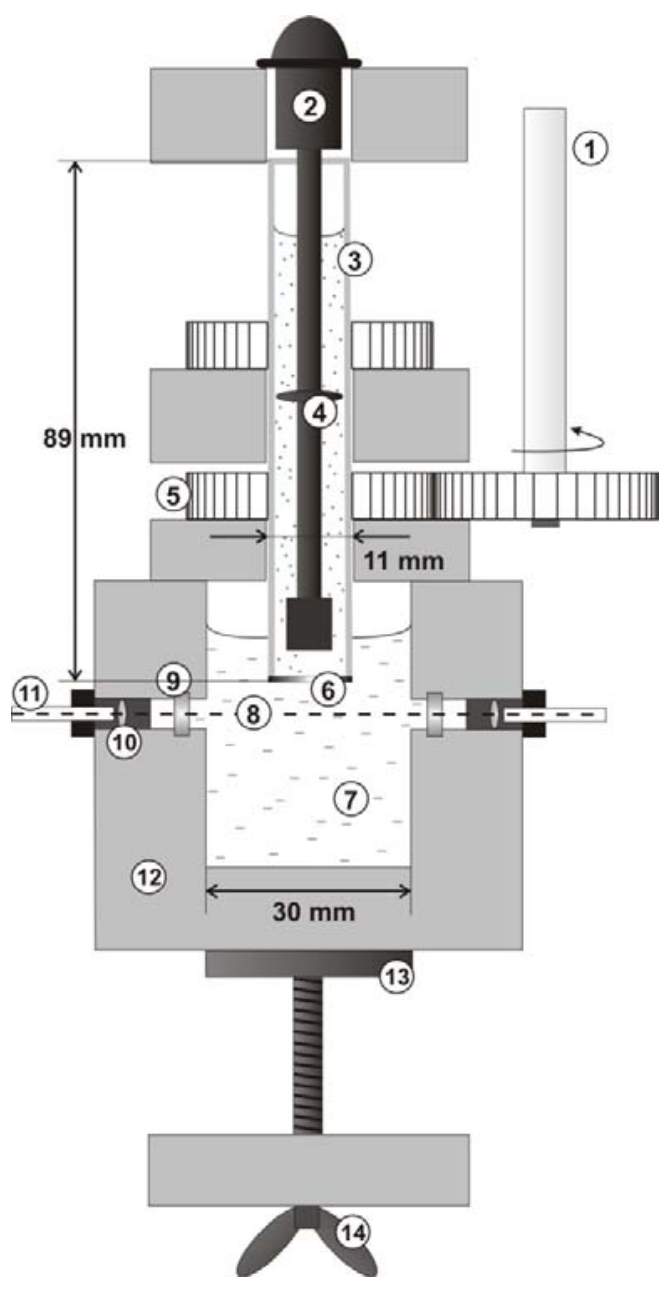

Figure 3.5. Schematic diagram of the permeation cell used for in situ UV measurements:

1. rotation controller

2. paddle

3. glass tube (donor compartment)

4. spacer disc

5. PTFE cogwheel tightly connected to the glass tube

6. PVDF membrane

7. acceptor solution,

8. UV source optical path

9. quartz window

10. quartz lens

11. fibre optic cable

12. PTFE acceptor cell

13. steel pad

14. steel pad screw [25]

\subsubsection{Hydrodynamics}

In some PAMPA implementations (see Table 3.3), the permeation study is carried out in a static environment where the compounds under investigation are allowed to diffuse from the donor to the acceptor wells over a period of 10-15 hours. Some PAMPA practitioners appear to follow these static experiment protocols. For the study of lipophilic molecules under these conditions, it is not uncommon to see an effective permeability of about $(15-30) \times 10^{-6} \mathrm{~cm} / \mathrm{s}$. It is now recognised that this is due to the resistance of unstirred water layers which becomes a dominant factor in measurement of effective permeability (see Equation 3.19). For instance, in an unstirred PAMPA assay, the UWL thickness can be as great as $2000-4000 \mu \mathrm{m}$ [23]. Since the UWL thickness in the human small intestine is assumed to be 30-100 $\mu \mathrm{m}$ [51], the unstirred permeation assay does not correctly match in vivo conditions. 


\section{Individual well stirring technique}

Early pioneering work by the Avdeef [24] and Faller [35] groups soon identified this issue, and attempted to reduce the thickness of the unstirred water layers by gentle shaking of the donor-acceptor sandwich. Apparently, shaking is not a very efficient method of agitation, as the acceptor wells are situated in a confined space, which hinders convection. The Avdeef group has subsequently reported an individual well stirring technique in plate-based permeability measurements [27]. The stirring speeds were reported to vary from $49-622 \mathrm{rpm}$. This technique has been evaluated using a set of drug molecules. The hydrodynamic analysis of stirred PAMPA measurements using Equation 3.22 revealed a value of 0.709 for the hydrodynamic exponent, $\alpha$.

\section{Rotating permeation cell}

Using the rotating permeation cell as shown in Figure 3.5, Velický et al. have carried out detailed permeation studies on several model drug molecules, including warfarin and verapamil under different donor $\mathrm{pH}$ values, concentrations and stirring rates [25]. Figure 3.6 shows the concentration $v s$. time plots and $\ln k v s$. time plots (Equation 3.17) of warfarin and verapamil. The slope, $a$, of the $\ln k$ time function where time $>\tau_{\mathrm{LAG}}$ was used to calculate the effective permeability, $P_{\mathrm{e}}$, using Equation 3.15. Here, the lag-time $\tau_{\mathrm{LAG}}$, was determined as follows: the In $k$ - time dependence data were fitted with a second order polynomial (usually within $0<$ time $<400 \mathrm{~s}$ ) and a linear function (usually time $>700 \mathrm{~s}$ ). The time

(a)

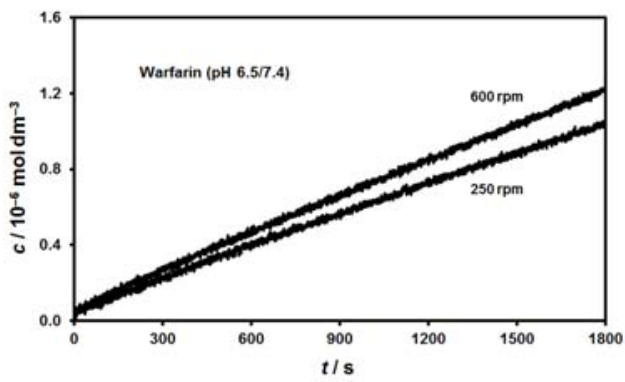

(b)

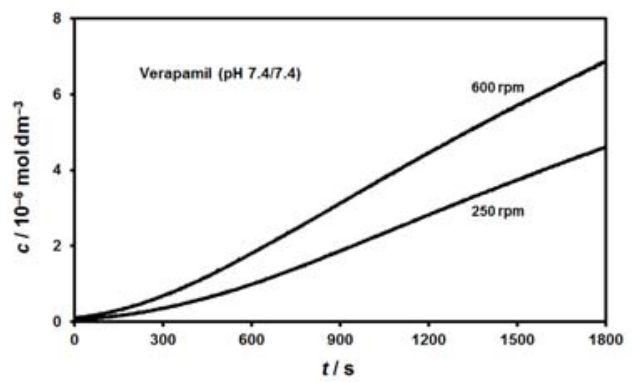

(c)

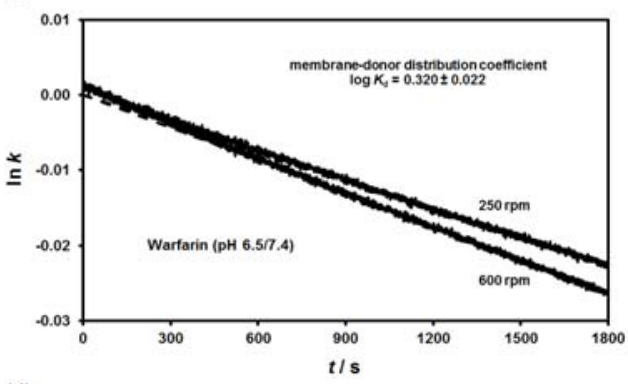

(d)

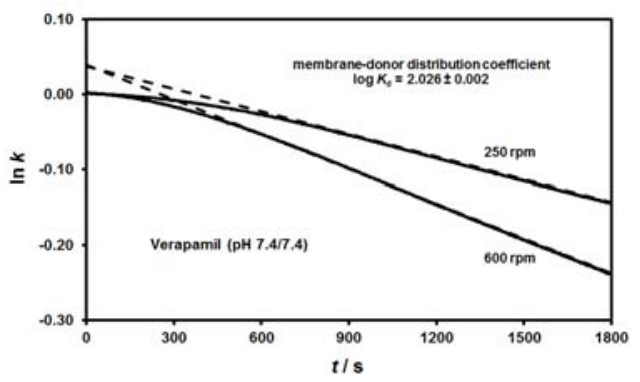

Figure 3.6. Example of concentration-time plots for (a) warfarin (309 nm) at donor/acceptor pH 6.5/7.4, (b) verapamil (280 $\mathrm{nm}$ ) at donor/acceptor $\mathrm{pH}$ 7.4/7.4. Derived ln $k$-time plots based on Equation 3.17 are shown for (c) warfarin and (d) verapamil [25] 
where the two fitted equations intersected was calculated as the lag-time $\tau_{\mathrm{LAG}}$. As shown in Figure 3.6c-d, warfarin exhibits a more linear $\ln k$ - time response than verapamil; the plot of the latter only becomes linear in the middle of the time course. The lag time for warfarin is very close to zero, but for verapamil, the lag times were determined as $1552 \mathrm{~s}$ and $1003 \mathrm{~s}$ at stirring rates of $250 \mathrm{rpm}$ and 600 $\mathrm{rpm}$, respectively. Given this unusual transient behaviour of verapamil, additional molecules were studied to gain further insight.

\section{Lag-time analysis}

Lag-time, $\tau_{\mathrm{LAG}}$, is regarded as the time at which the permeation system, consisting of the donor, membrane and acceptor, reaches a steady-state and the permeation is driven only by the solute concentration gradient between the bulk donor and acceptor. Mathematically, in the initial transient period, permeation follows a complex pattern of solute partitioning to the membrane and diffusion according to Fick's second law:

$$
\frac{\partial c}{\partial t}=D \frac{\partial^{2} c}{\partial x^{2}}
$$

where $c$ is the solute concentration, $t$ is time, $x$ is the position and $D$ is the diffusion coefficient (aqueous and/or in the membrane). After time $t=\tau_{\mathrm{LAG}}$ when the steady-state across the donor-membrane-acceptor system has been established, the permeation can be approximated by Fick's first law:

$$
J=-D \frac{\partial c}{\partial x}
$$

where $J$ is the diffusive flux and the other symbols have the same meaning as indicated above. As the system reaches the steady-state, $\ln k$ becomes linear with time. Before the steady-state is reached, ln $k$ shows a non-linear dependence on time which can be approximated with a parabolic function. The ln $k v s$. time plots of four drug molecules, including propranolol, midazolam, quinine and verapamil, are shown in Figure 3.7. It can be seen that the lag-time of verapamil is longer than the actual time of measurement. Careful examination of the permeation profile shows that the steady-state was not completely reached within the 20 minute experiment. Reports on in vitro permeation across lipid bilayers and supported liquid membranes have quoted lag-time values in the range of 1-30 min [52-53]. As shown in Table 3.4, the lag-times of propranolol, midazolam, quinine and verapamil as determined in this study are consistent with the literature. It has been reported that the intestinal residence time is generally between 1-3 $\mathrm{h}$ [54]. For the drug molecules investigated in this study, the lagtimes are well within the intestinal residence time, suggesting that the initial transient phase of permeation is unlikely to be an issue hindering drug absorption in vivo. However, for lipophilic molecules where the doses are low, the expected longer lag-times could be an issue and might potentially lead to unexpectedly poor absorption in pre-clinical and/or clinical studies. 


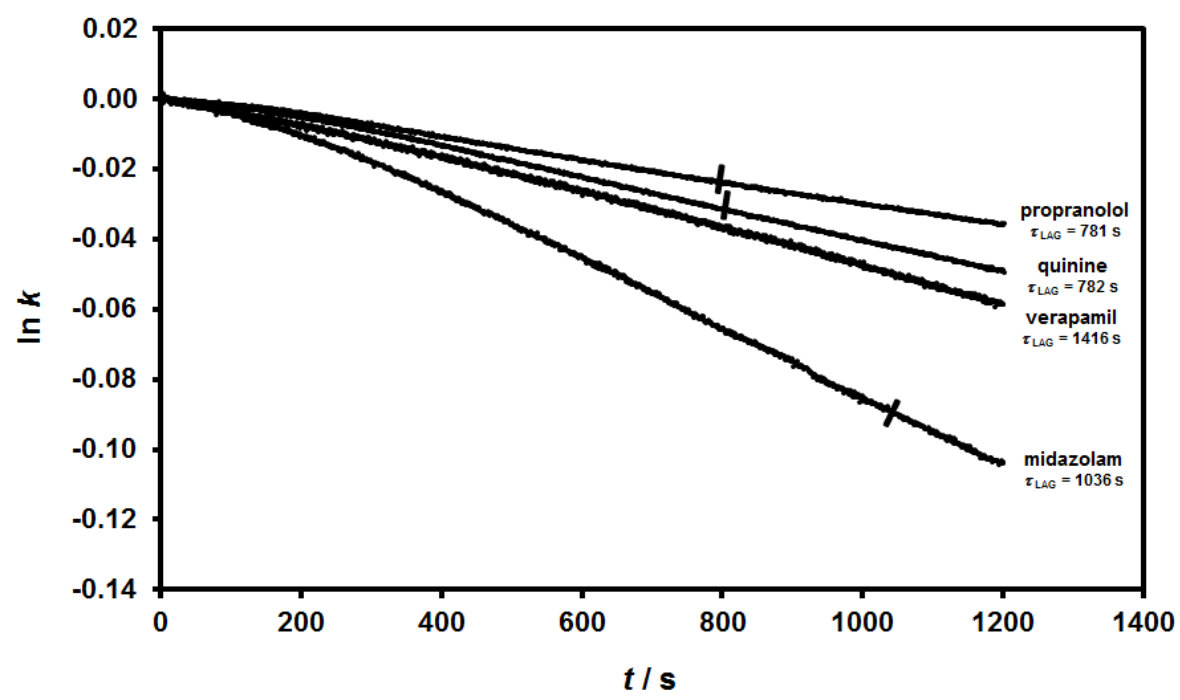

Figure 3.7. Permeation ln $k$ - time plots of propranolol, quinine, verapamil and midazolam (donor/acceptor $\mathrm{pH} 6.5 / 7.4$ and stirring rate $280 \mathrm{rpm}$ ) showing the different lag-times as vertical bars. The initial donor concentrations were 320,255 , 210 and $80 \mu \mathrm{M}$ for propranolol, quinine, verapamil and midazolam, respectively

Table 3.4. Lag-time and physicochemical properties of propranolol, quinine, midazolam and verapamil.

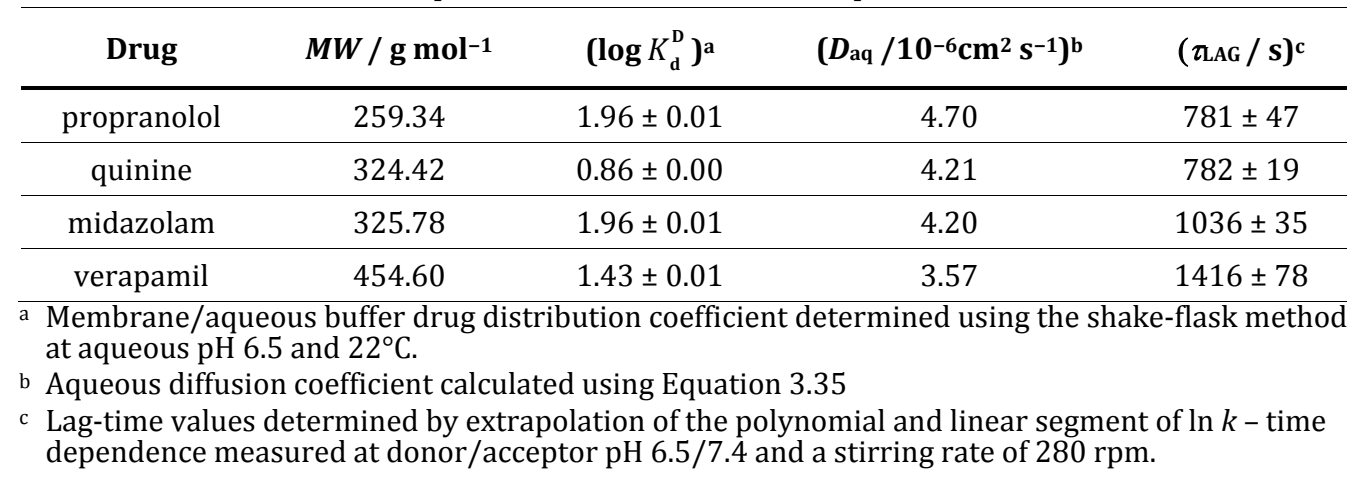

\section{Lag-time dependence on stirring rate}

Stirring decreases the unstirred water layer thickness and thus increases the measured effective permeability. Figure 3.8 shows the ln $k$ time profiles of propranolol obtained at stirring rates of $60,80,110,160$ and $280 \mathrm{rpm}$. Similar to the permeability terms, there are two separate components contributing to the overall lag-time value. One component, $\tau_{\mathrm{LAG}, \mathrm{m}}$, arises from the partitioning and loading of a solute to the membrane, whereas the other term, $\tau_{\mathrm{LAG}, \mathrm{u}}$, arises due to solute transport through the unstirred water layer adjacent to both sides of the membrane. 


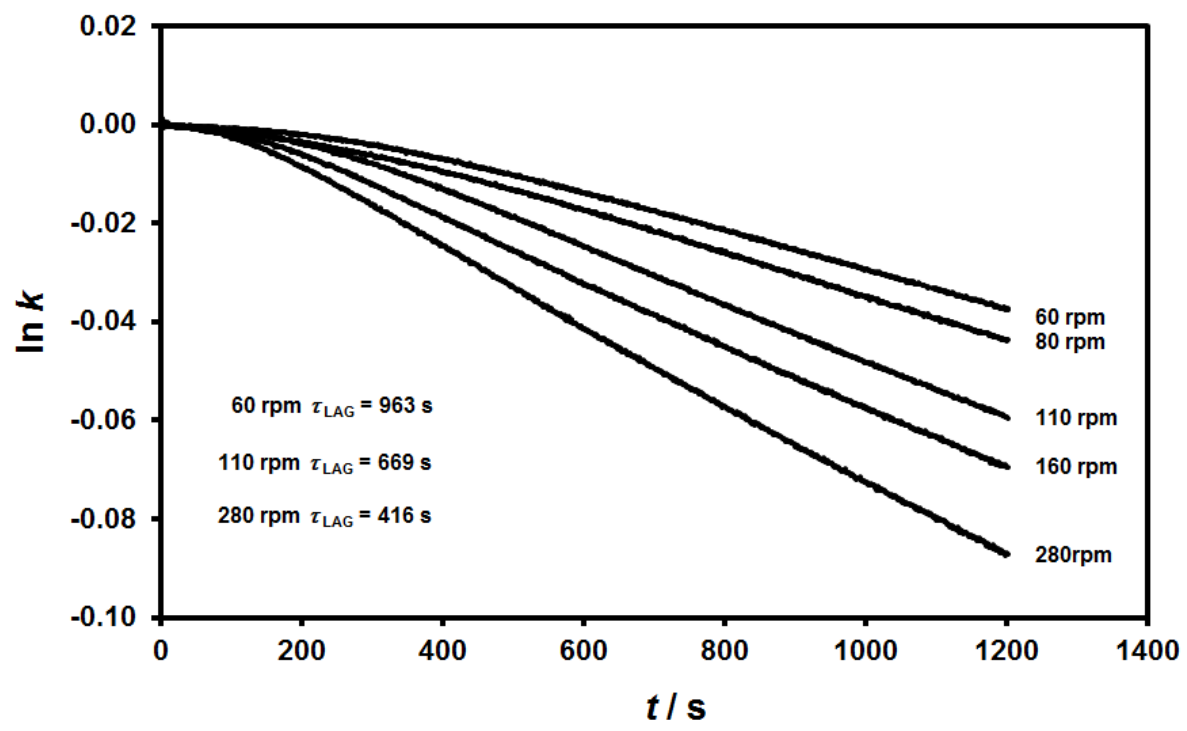

Figure 3.8. Permeation $\ln k$ - time plots of propranolol (225 nm) at donor/acceptor $\mathrm{pH} 7.4 / 7.4$

The observed lag-time, $\tau_{\mathrm{LAG}}$, is a sum of the two, which can be described by the following equation:

$$
\tau_{\mathrm{LAG}}=\tau_{\mathrm{LAG}, \mathrm{u}}+\tau_{\mathrm{LAG}, \mathrm{m}}
$$

For the membrane loading process, the lag-time can be approximated from Fick's second law as [55-56]:

$$
\tau_{\mathrm{LAG}, \mathrm{m}}=\frac{h^{2}}{6 D_{\mathrm{m}}}
$$

where $h$ represents membrane thickness and $D_{\mathrm{m}}$ membrane diffusion coefficient. Using a similar relationship, where thickness and the membrane diffusion coefficient are replaced with UWL thickness, $\delta_{\mathrm{u}}$, and the aqueous diffusion coefficient, $D_{\text {aq }}$, for the UWL dependent term, the following relationship is obtained:

$$
\tau_{\mathrm{LAG}}=K_{\tau} \frac{\delta_{u}^{2}}{D_{\mathrm{aq}}}+\frac{h^{2}}{6 D_{\mathrm{m}}}
$$

where $K_{\tau}$ is an empirical constant. The aqueous term in Equation 3.31 can be further expressed as a function of the stirring rate using Equation 3.32:

$$
\tau_{\mathrm{LAG}}=2.6 K_{\tau} D_{\mathrm{aq}}{ }^{-1 / 3} v^{1 / 3} \omega^{-1}+\frac{h^{2}}{6 D_{\mathrm{m}}}
$$

Lag-time values measured for propranolol permeation at several different stirring rates are plotted in Figure 3.9. The graph shows the linear dependence of 
$\tau_{\mathrm{LAG}}$ on the inverse angular velocity of stirring, $1 / \omega$, for propranolol at donor/acceptor $\mathrm{pH}$ 6.5/7.4. According to Equation 3.32, the intercept of this dependence should be equal to $\tau_{\mathrm{LAG}, \mathrm{m}}$. Substituting the membrane thickness (assumed to be 125-200 $\mu \mathrm{m}$ due to excess organic solvent immobilised on the membrane filter) and the membrane diffusion coefficient (calculated from Equation 3.12 and averaged over the values measured at different donor $\mathrm{pH}$ 5.510.5 ) to Equation 3.30 yields a membrane lag-time values, $\tau_{\mathrm{LAG}, \mathrm{m}}$, in the range 113-290 s. This is in a good agreement with the experimental value of $236 \pm 2 \mathrm{~s}$ (Figure 3.9).

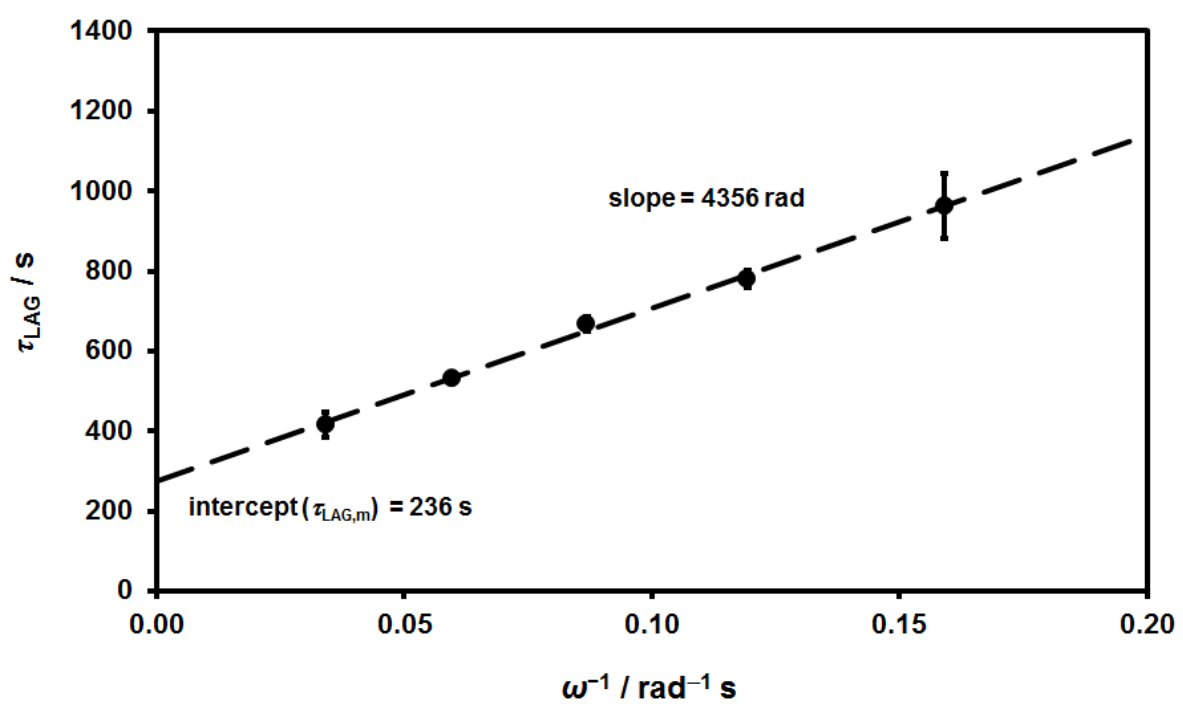

Figure 3.9. Dependence of the lag-time on the inverse angular velocity of stirring for propranolol permeation at donor/acceptor $\mathrm{pH}$ 6.5/7.4

\section{Lag-time dependence on the concentration gradient}

Figure 3.10 shows the effects of the concentration gradient on lag-time for permeation studies on propranolol. The experiments were carried out at donor/acceptor $\mathrm{pH} 7.4 / 7.4$ and stirring rates of 60, 110 and $280 \mathrm{rpm}$. The initial drug concentration in the donor solution, $c_{\mathrm{D}}(0)$, was varied from 30 to $300 \mu \mathrm{M}$. As shown in Figure 3.10, the lag-time was found to decrease with an increase in the concentration gradient between the donor and acceptor phase. It is apparent that a larger concentration gradient generates a larger driving force for mass transport and therefore leads to a shorter lag-time to reach the steady-state. This observation underlines the importance of unified inter-laboratory permeation assay standards in cases where the analytical permeability model does not allow for the lag-time. 


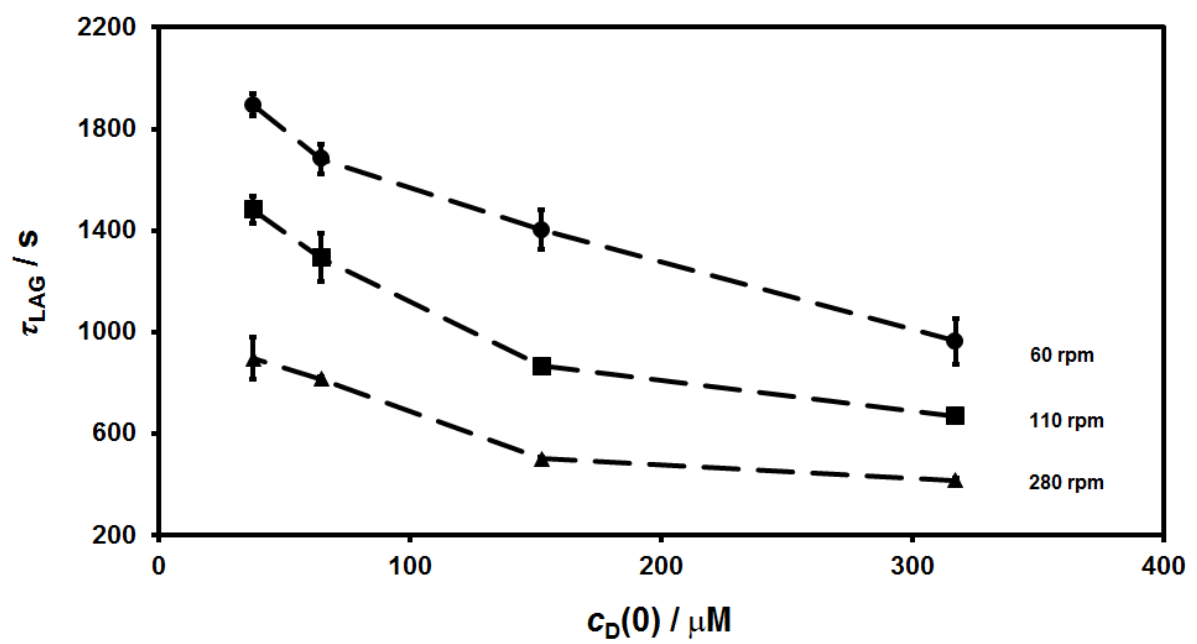

Figure 3.10. Lag-time dependence on the initial donor concentration of propranolol at donor/acceptor $\mathrm{pH}$ 7.4/7.4

\section{Hydrodynamic analysis}

Figure 3.11 shows the hydrodynamic analysis on effective permeability as a function of the stirring rate (Equation 3.24) for warfarin and verapamil. Our data revealed that, for warfarin, the ideal hydrodynamic exponent, $\alpha=0.5$, provides a good fit, while for verapamil, an $\alpha$ value of 1 is required. It was noted that the hydrodynamic exponent $\alpha$ describes the permeation sensitivity to stirring. With lower values of $\alpha$, transport becomes membrane-limited; conversely, a higher $\alpha$ value implies diffusion-limited permeation, and is sensitive to stirring. We have extended the study to a larger set of 31 drug molecules. As shown in Figure 3.12, $\alpha$ correlates with the lipophilicity of the molecule (the membrane/buffer distribution coefficient at $\mathrm{pH}$ 6.5) via the following empirical polynomial equation:

$$
\alpha=0.0125\left(\log K_{\mathrm{d}}\right)^{3}+0.0424\left(\log K_{\mathrm{d}}\right)^{2}+0.0732 \log K_{\mathrm{d}}+0.0905
$$

where $K_{\mathrm{d}}$ represents the membrane/buffer distribution coefficient at pH 6.5.

\section{Permeability-pH profiles}

Figure 3.13 shows the permeability-pH profiles of warfarin and verapamil. Permeation data obtained under unstirred conditions were included (filled black square symbols in Figure 3.13). The intrinsic permeability coefficient $\left(P_{0}\right)$ was calculated by least-squares analysis for all the stirring rates shown $(0,250,400$, 600, 1000 and $1500 \mathrm{rpm}$ ) via Equation 3.26. The intrinsic permeability was approximately constant for all stirring rates, as expected, and the average value is plotted as a dashed line. The hydrodynamic membrane permeability coefficient extrapolated from the dependence of the effective permeability on stirring rate via Equation 3.24 was plotted for each $\mathrm{pH}$ as a filled black diamond symbol. This is consistent with the membrane permeability coefficient, $P_{\mathrm{m}}$, calculated from the 
(a) Effective permeability vs. stirring rate - warfarin at $\mathrm{pH} 6.5 / 7.4$

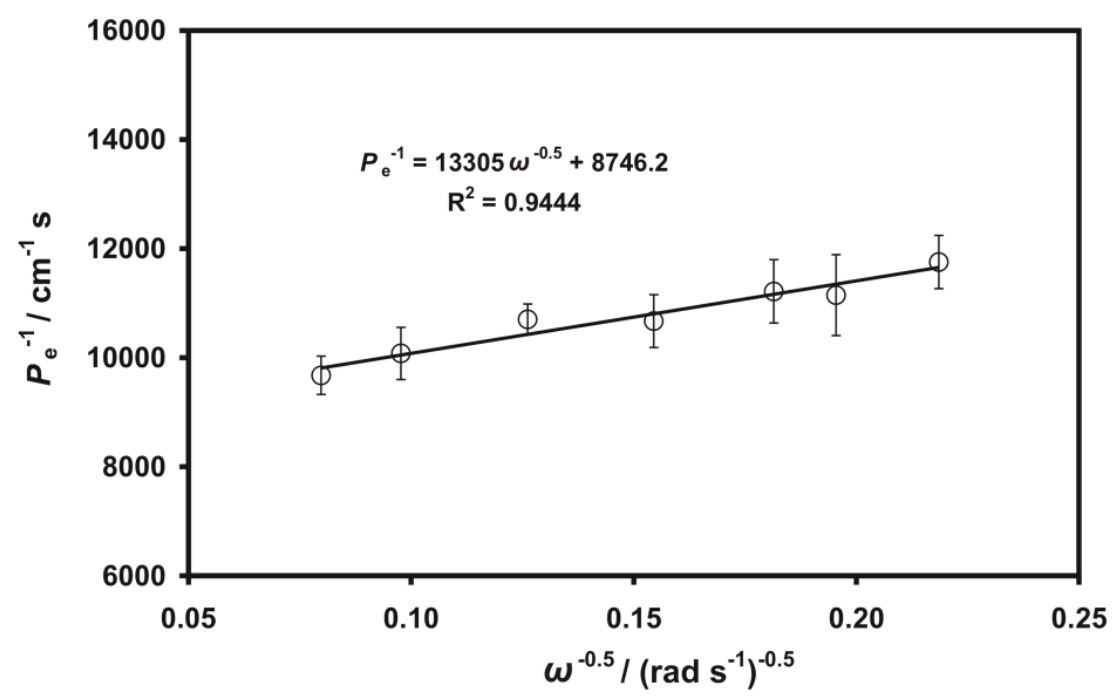

(b) Effective permeability vs. stirring rate - verapamil at $\mathrm{pH} 7.4 / 7.4$

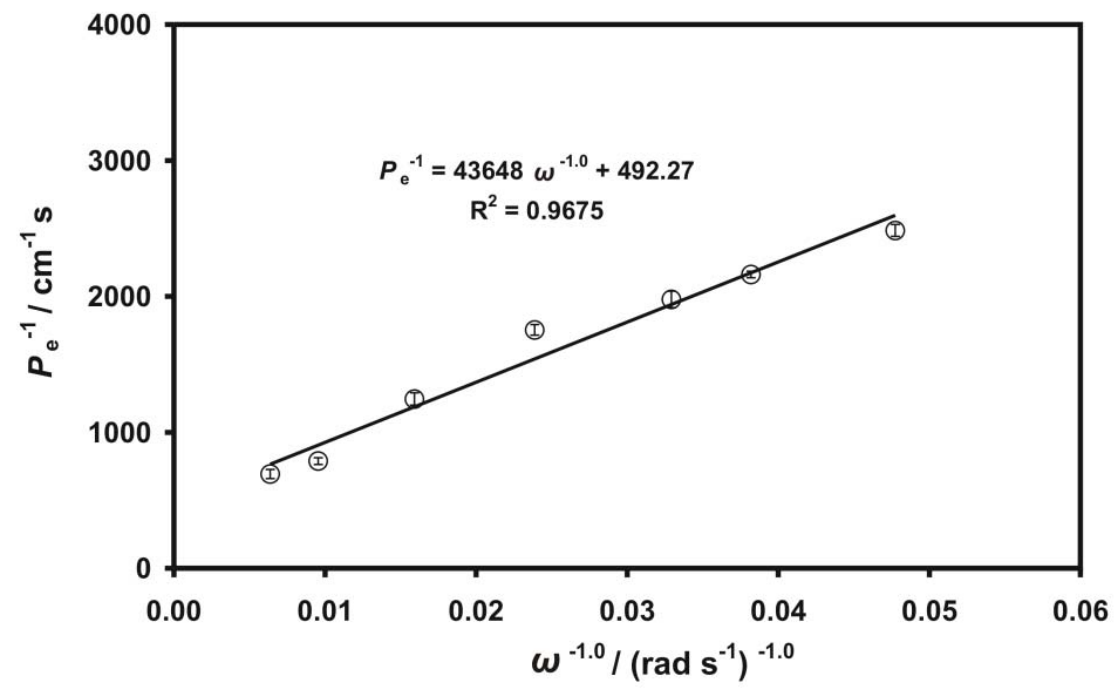

Figure 3.11. Dependence of the inverse of effective permeability on the stirring rate for (a) warfarin at $\mathrm{pH} 6.5 / 7.4$, (b) verapamil at $\mathrm{pH} 7.4 / 7.4$. The warfarin and verapamil effective permeability values are plotted against $\omega^{-0.5}$ and $\omega^{-1.0}$, respectively [25]

$P_{0}$ value using Equation 3.25 (plotted as a solid curve). Finally, the hydrodynamic UWL permeability coefficient, $P_{\mathrm{u}}$, was calculated separately for each stirring rate and pH using Equation 3.19 (dashed curves in Figure 3.13). 


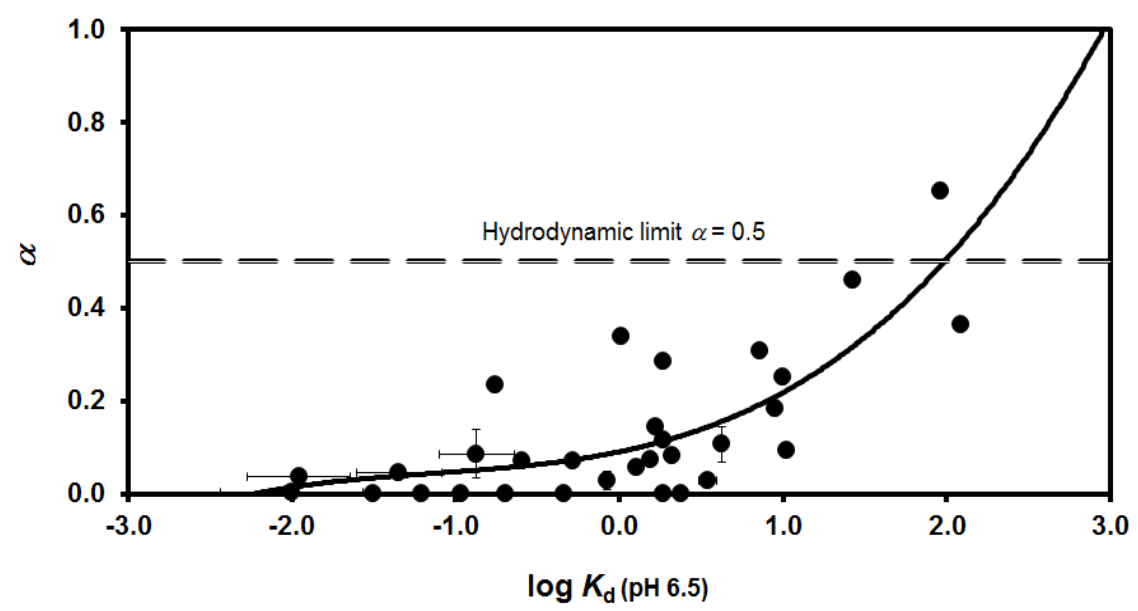

Figure 3.12. Dependence of $\alpha$ on the membrane/buffer distribution coefficient for the pH 6.5/7.4 permeation experiment of 31 studied drug molecules. The 31 drug molecules are: acetaminophen, antipyrine, atenolol, betamethasone, cefixime, cephalothin, cetirizine, chlorpheniramine, chlorthalidone, colchicines, diclofenac, eprosartan, fexofenadine, gatifloxacin, metolazone, midazolam, nafcillin, naproxen, norfloxacin, oxybutynin, pindolol, propranolol, pyridoxine, quinine, risperidone, salicylic acid, theophylline, tolbutamide, verapamil, warfarin and zopiclone [65]

Warfarin is a weak acid, with $\mathrm{p} K_{\mathrm{a}}=4.82$ [23]. In $\mathrm{pH}$ range 6.5-8.0, it is predominantly ionised, and shows little change in effective permeability according to stirring rate (Figure 3.13a). This implies that permeation is membrane-limited and the UWL is very thin, giving little resistance to drug diffusion. In the $\mathrm{pH}$ range of 3.5-6.5, the permeability separation with stirring rate increases and permeation becomes diffusion-limited, largely affected by the increasing UWL thickness. In contrast, verapamil is a weak base with $\mathrm{p} K_{\mathrm{a}}=9.07$ [23]. As shown in Figure 3.13b, the effective permeability is more dependent on stirring rate, and is much higher across the entire $\mathrm{pH}$ range than that of warfarin. The difference between the unstirred permeability value and membrane permeability coefficient is more than $1.7 \log$ units at a donor $\mathrm{pH}$ of 6.5. In other words, the UWL thickness under these conditions is estimated to be $3830 \mu \mathrm{m}$ (using Equations 3.19 and 3.23, and $D_{\text {aq }}$ is given in Table 3.4), which yields a UWL resistance far greater than that of the membrane. This suggests that the determination of effective permeability using a static PAMPA method would lead to problematic results, and precise control of hydrodynamics is essential to delineate the effects of UWL. 
(a)

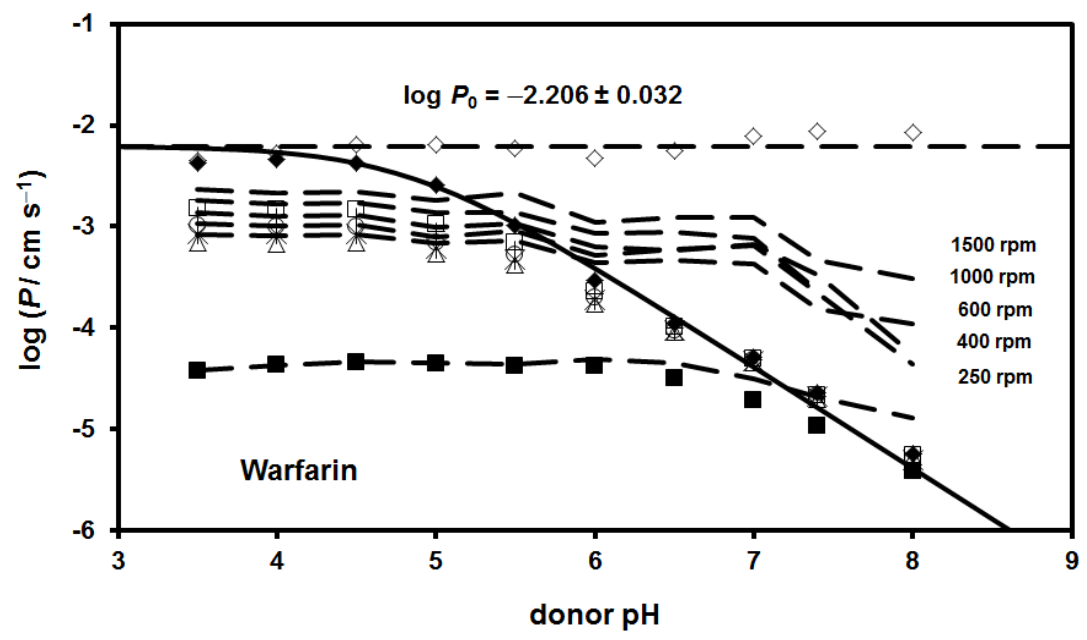

(b)

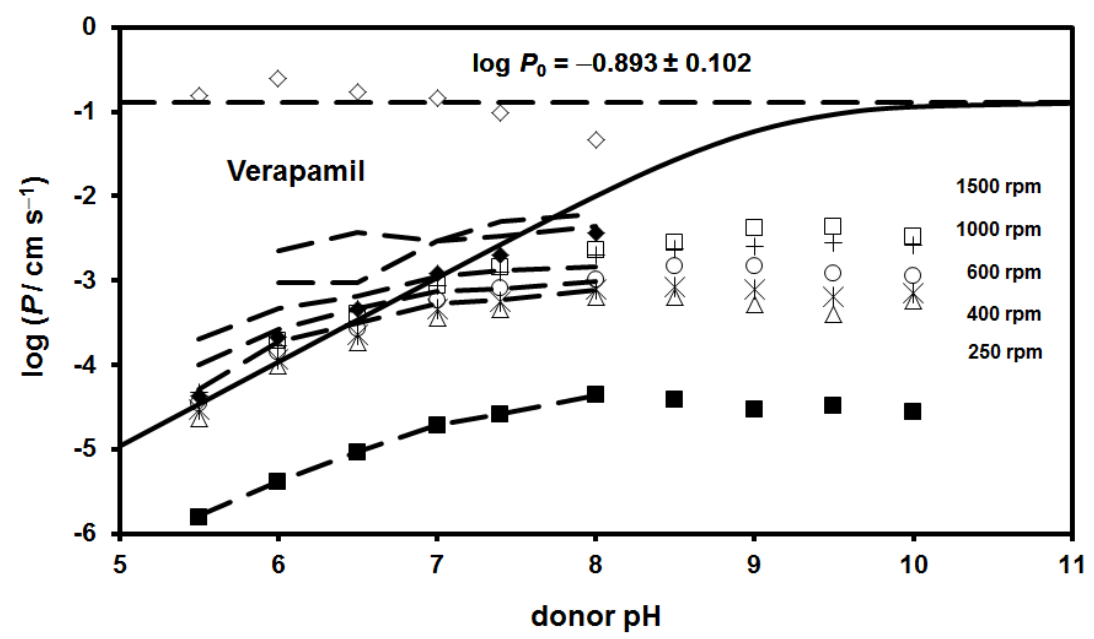

Figure 3.13. Permeability-pH profiles of a) warfarin and (b) verapamil [25]

\subsection{APPLICATIONS OF PAMPA DATA TO PREDICT AND/OR MODEL IN VIVO DATA}

As described before, PAMPA permeability data have been applied for the prediction of percent absorbed in humans (Sections 3.3.1.1, 3.3.1.4-3.3.1.8). In these studies, marketed drugs with a known percent absorbed in humans were investigated in the respective lipid models. The permeability data were reported to be correlated with the data on the percent absorbed in humans. Except for the DOPC (Section 3.3.1.2) [24], DS-PAMPA (Section 3.3.1.3) [26,36-37], $n$-hexa- 
decane (Section 3.3.1.4) [35], immobilised phospholipid vesicles (Section 3.3.1.7) [45] and lipid/cholesterol/octanol (Section 3.3.1.8) [46] models, permeation studies were carried out without agitation. Readers are directed to the relevant publications for further details.

The presence of the unstirred water layer in a PAMPA study could have a profound effect on the accuracy of the data, particularly for lipophilic molecules. In Section 3.3.2.3, we described the rotating permeation cell and the individual well stirring technique, which offer precise control of the hydrodynamics in the donor/acceptor compartments. Moreover, we have discussed approaches (Section 3.2) to disentangle the unstirred water layer permeability $\left(P_{\mathrm{u}}\right)$ from the effective permeability $\left(P_{\mathrm{e}}\right)$. In this section, we will focus our attention on these two techniques to build correlations between in vitro permeability and the absorbed fraction of drugs in humans.

A simple passive diffusion permeability model would not be expected to accurately predict the intestinal absorption of smaller hydrophilic drugs, since the paracellular route of absorption would not be adequately addressed. Sugano et al. [41] reported the use of a calculated contribution based on the Renkin function [42] to account for transport via the paracellular route. In the present study, this approach will be utilised to enhance the permeability data, and will be discussed in Section 3.4.1. The plug flow absorption model as developed by Amidon et al. [57] will be used to correlate the in vitro permeability (corrected for paracellular transport and unstirred water layer permeability) and the data on the absorbed fraction in humans (Section 3.4.2).

In Section 3.4.3, the rotating permeation cell will be applied to study a set of 26 marketed drugs (acidic, basic and neutral drugs). In Section 3.4.4, the individual well stirring technique in conjunction with the DS-PAMPA model will be applied to study a set of 33 amphoteric drugs. Drugs where the first pass hepatic clearances are low to moderate, with published human absolute bioavailability data spanning low to high values, were deliberately selected in these studies. In the modelling of the data, the absorbed fraction was used, which is derived from human bioavailability data corrected for first pass hepatic clearance, to avoid erroneous correlation with permeability. Care was taken to include drugs with moderate to good aqueous solubility to ensure that the absorption process was not solubility limited. In other words, the selected drug molecules generally fall within BCS classes I and III. These conditions provided the basis for a rigorous assessment of the correlation between the in vitro drug permeability coefficient and literature bioavailability determined in vivo.

\subsubsection{Corrections for paracellular transport}

Adson et al. $[19,28]$ have proposed a general expression for paracellular permeability, which is written as follows: 


$$
P_{\mathrm{p}}=\left(\frac{\varepsilon}{\delta}\right) \cdot D_{\mathrm{aq}} \cdot F\left(\frac{r}{R}\right) \cdot\left[f_{( \pm / 0)}+f_{(+)} \cdot \frac{\kappa \cdot|\Delta \phi|}{1-e^{-\kappa|\Delta \phi|}}+f_{(-)} \cdot \frac{\kappa \cdot|\Delta \phi|}{e^{+\kappa|\Delta \phi|}-1}\right]
$$

where $(\varepsilon / \delta)$ represents the porosity-pathlength ratio (the porosity, $\varepsilon$, is the relative surface area of the junction opening divided by the total epithelial surface area, and the pathlength, $\delta$, represents the thickness of the restrictedjunction domain times the tortuosity of the paracellular route). $D_{\text {aq }}\left(\mathrm{cm}^{2} \mathrm{~s}^{-1}\right)$, is the aqueous diffusivity, which was estimated at $25{ }^{\circ} \mathrm{C}$ using the empirical function based on molecular weight as follows [23,58-60]:

$$
\log D_{\mathrm{aq}}=-4.14-0.46 \log M W
$$

$F(r / R)$ is the Renkin molecular sieving function, which is defined as [42]:

$$
F\left(\frac{r}{R}\right)=\left(1-\left(\frac{r}{R}\right)\right)^{2} \times\left(1-2.104\left(\frac{r}{R}\right)+2.09\left(\frac{r}{R}\right)^{3}-0.95\left(\frac{r}{R}\right)^{5}\right)
$$

The symbol $r$ is the solute hydrodynamic radius and $R$ is the pore radius, which was calculated using the Sutherland-Stokes-Einstein spherical-particle equation [58]:

$$
r=\left(0.92+\frac{21.8}{M W}\right) \frac{k_{\mathrm{B}} T}{6 \pi \eta D_{\mathrm{aq}}}
$$

where $k_{\mathrm{B}}$ is the Boltzmann constant, $T$ is the absolute temperature, $\eta$ is the dynamic viscosity of the solvent $\left(0.00893\right.$ poise for water at $\left.25^{\circ} \mathrm{C}\right)$.

The square-bracket term in Equation 3.34, which is referred as $E(\Delta \varphi)$, is a function of the electrical potential drop, $\Delta \varphi(\mathrm{mV})$, across the junction created by negatively-charged residues (carboxylate and phosphate) lining the junctional pores, where $f( \pm / 0), f(+)$ and $f(-)$ are the concentration fractions of the neutral/zwitterionic, cationic and anionic forms of the drug, respectively. $\kappa=$ $F /\left(k_{\mathrm{B}} T N_{\mathrm{A}}\right)$, where $N_{\mathrm{A}}$ is Avogadro's number and $F$ is the Faraday constant. The term in the brackets, $E(\Delta \varphi)$, is 1 for neutral molecules, about 1.4 for cations and 0.7 for anions. In the present study, the parameters $R=12.9 \AA, \varepsilon / \delta=0.78 \mathrm{~cm}^{-1}$ and $\Delta \varphi=-30 \mathrm{mV}$ were taken from Avdeef [58] who derived these parameters by a re-analysis of the Caco-2 permeability data of several paracellular markers published by Adson et al. [19].

The membrane permeability, $P_{\mathrm{m}}$, obtained from the in vitro permeation study (from Equation 3.19 and Equations 3.22 or 3.23, depending on the experimental technique used) is corrected for paracellular transport (using Equation 3.34) as follows:

$$
\frac{1}{P_{\mathrm{e}}^{\mathrm{INT}}}=\frac{1}{P_{\mathrm{u}}}+\frac{1}{S \times P_{\mathrm{m}}+P_{\mathrm{p}}}
$$

where $P_{\mathrm{e}}{ }^{\mathrm{INT}}$ and $P_{u}$ represent, respectively, the effective permeability (corrected for paracellular transport) and the permeability term due to the unstirred water 
layer permeability adjacent to the intestinal epithelial layer. $P_{\mathrm{p}}$ is calculated by Equation 3.34 , while $P_{\mathrm{u}}$ is calculated using Equation 3.23 by assuming an unstirred water layer thickness $\left(\delta_{\mathrm{u}}\right)$ of $200-500 \mu \mathrm{m}$ [61-62]. The variable $S$ is a scaling factor. The choice of the scaling factor depends on the particular permeability assay/paracellular model used, and could be treated as an adjustable parameter, with its optimum value found from the best fit of the effective permeability and fraction absorbed data in the plug-flow model, as described in Section 3.4.2.

\subsubsection{Absorption model}

From the literature on absolute oral bioavailability data, and assuming that gut wall metabolism is negligible (i.e. $F_{\mathrm{g}} \approx 1$ ), the fraction absorbed $F_{\mathrm{a}}$ can be calculated as follows (see Equation 3.2):

$$
F_{\mathrm{a}} \approx \frac{F}{F_{h}}
$$

where $F_{\mathrm{h}}=1-C L_{\mathrm{h}} / Q_{\mathrm{h}}$. The symbols $C L_{\mathrm{h}}$ and $Q_{\mathrm{h}}$ represent, respectively, first pass hepatic clearance [63] and hepatic blood flow (set at $23 \mathrm{~mL} \mathrm{~min}-1 \mathrm{~kg}^{-1}$ body weight [2]). To evaluate the correlation between the effective permeability $\left(P_{\mathrm{e}}^{\mathrm{INT}}\right)$ and the fraction absorbed, the $\% F_{\mathrm{a}}$ data are fitted against the plug-flow absorption model [57-62,64]:

$$
\% F_{\mathrm{a}}=H\left(1-\exp \left(-G P_{\mathrm{e}}^{\mathrm{INT}}\right)\right)
$$

where $G$ represents the Graetz number (a dimensionless number which describes laminar flow in the intestine, treated as a tube, in the plug flow model). In the present study, $G$ and $H$ are regarded as fitting constants.

\subsubsection{Correlation between in vitro permeability and the absorbed fraction of drugs in humans}

Figure 3.14 shows the absorbed fraction of the 26 drug molecules as a function of the effective permeability $\left(\log P_{\mathrm{e}}^{\mathrm{INT}}\right)$, with the solid curve representing the best fit to Equation 3.40 [65]. The optimised parameters are: $H=100, G=2.14 \times 10^{6} \mathrm{~cm}^{-1} \mathrm{~s}$ and $S=0.014$. The dotted curves are derived from estimated errors in the log $P_{\mathrm{e}}^{\mathrm{INT}}$ values with an average value of 0.35 , which is estimated based on the lab-tolab variability of the Caco-2 paracellular parameters [58]. For the upper dashed curve, the $H$ parameter is 100 , while a value of 85 is used for the lower dashed curve (in part to reflect the estimated errors in the $\% F$ data). The estimated errors in the $\% F_{\text {a }}$ data were determined as a range of values spread over different published $\% F$ values. For those molecules where errors in the published $\% F$ values were not available, the average error value of $9 \%$ was used. As shown in Figure 3.14, the absorbed fraction data of these molecules were generally well predicted, within experimental uncertainty, by the approach described in this chapter. 


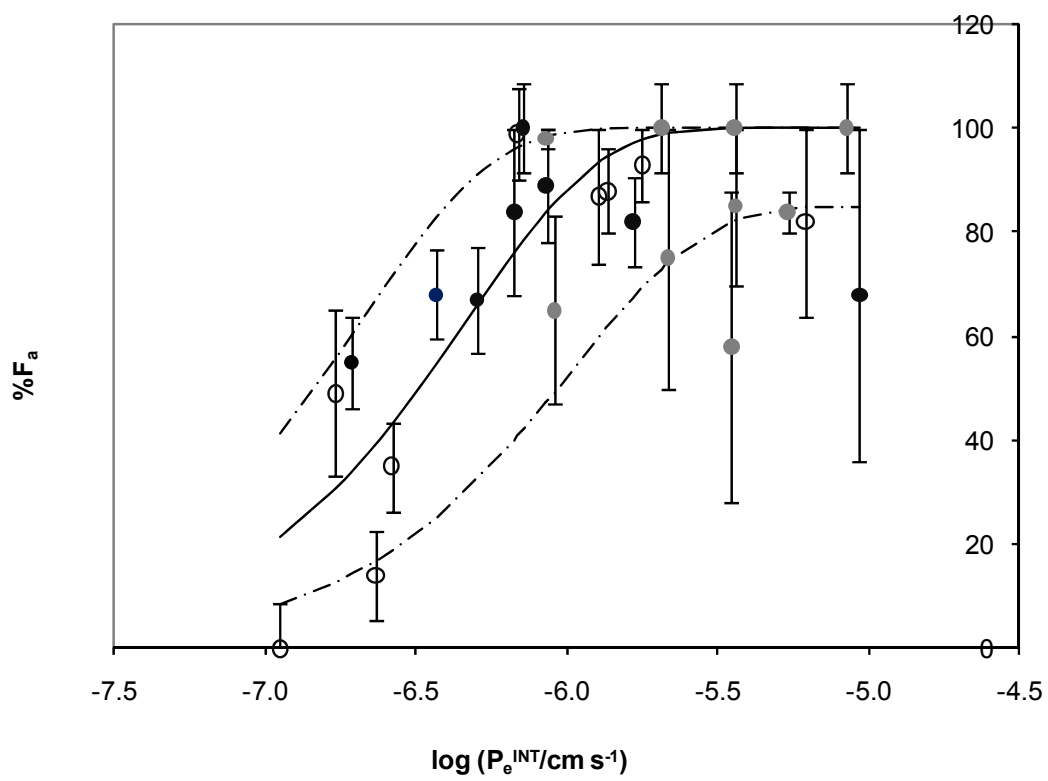

Figure 3.14. Correlation between fraction absorbed $\left(\% F_{a}\right)$ and effective permeability (corrected for paracellular transport and unstirred water layer permeability (UWL thickness $=200 \mu \mathrm{m}$ )). The solid curve is the best fit of the function represented by Equation 3.40. The dashed curves are based on estimated errors in the $\log P_{\mathrm{e}}{ }^{\text {INT }}$ values (0.35). Unfilled circle - acid; grey circle - base; black circle - neutral.

The 26 drugs used are: acetaminophen, antipyrine, atenolol, betamethasone, cefixime, cephalothin, chlorpheniramine, chlorthalidone, colchicine, diclofenac, eprosartan, metolazone, midazolam, nafcillin, naproxen, oxybutynin, pindolol, propranolol, quinine, risperidone, salicylic acid, theophylline, tolbutamide, verapamil, warfarin and zopiclone.

The average absolute bioavailability data were taken from several sources [68-72] and corrected for hepatic clearance $[63,72]$ to generate the $\% F_{a}$ data [65]

\subsubsection{Prediction of the absorbed fraction of amphoteric drugs in humans}

Given the charge/polar nature of zwitterionic drugs, the permeability across an artificial membrane with low lipid content could be low. It was felt that the $20 \%$ phospholipid content in the DS-DAMPA method would facilitate the permeation of charged species by offering a compensating source of hydrogen bonding within the membrane, which may provide a more sensitive measure of the permeation rates. Table 3.5 lists the $P_{\mathrm{e}}^{\mathrm{INT}}$ values for selected amphoteric drugs deduced at donor $\mathrm{pH} 6.5$ using the DS-DAMPA model, in conjunction with individual well stirring [39]. The effective permeability values ranges from $0.2 \times 10^{-6}$ (ceftazidime) to $84 \times 10^{-6} \mathrm{~cm} \mathrm{~s}^{-1}$ (melphalan), with a mean value of $14 \times 10^{-6} \mathrm{~cm} \mathrm{~s}^{-1}$. As shown in Table 3.5, about a third of the drugs were predicted to permeate the intestinal barrier predominantly by the paracellular route, with acyclovir, ceftazidime, daunorubicin, famotidine, ganciclovir, levocarnithine and terbutaline almost entirely transported via the paracellular route, but with a low average absorbed fraction of less than $20 \%$. 
Table 3.5. Effective permeability, $P_{\mathrm{e}}{ }^{\mathrm{INT}}$, percent contribution of the paracellular component, Para, and the fraction absorbed data of the 33 selected amphoteric drug molecules, $F_{\mathrm{a}}$

\begin{tabular}{|c|c|c|c|c|}
\hline Drug & $\begin{array}{c}P \mathrm{e}^{\mathrm{INT}}(\mathrm{pH} \mathrm{6.5}) \\
10^{-6} \mathrm{~cm} \mathrm{~s}^{-1} \\
\end{array}$ & Para, \% & MW & $\begin{array}{l}\text { Fraction absorbed } \\
\text { in human }\left(F_{\mathrm{a}} / \%\right)^{\mathrm{a}}\end{array}$ \\
\hline Acyclovir & 0.8 & 99 & 225.20 & $26 \pm 9$ \\
\hline Amdinocillin & 0.9 & 46 & 325.40 & $14 \pm 7$ \\
\hline Benazepril & 17 & 1 & 424.49 & $37 \pm 7$ \\
\hline Ceftazidime & 0.2 & 96 & 546.60 & $0 \pm 7$ \\
\hline Cerivastatin & 15 & 1 & 459.55 & $68 \pm 16$ \\
\hline Cetirizine & 42 & 0 & 388.89 & $93 \pm 7$ \\
\hline Chlortetracycline & 3.7 & 5 & 478.88 & $65 \pm 9$ \\
\hline Ciprofloxacin & 2.8 & 18 & 331.34 & $100 \pm 7$ \\
\hline Daunorubicin & 0.3 & 99 & 527.50 & $0 \pm 7$ \\
\hline Demeclocycline & 3.8 & 4 & 464.85 & $66 \pm 7$ \\
\hline Doxycycline & 20 & 1 & 444.43 & $95 \pm 7$ \\
\hline Enoxacin & 7.7 & 6 & 320.32 & $100 \pm 7$ \\
\hline Famotidine & 0.6 & 98 & 337.45 & $58 \pm 7$ \\
\hline Fexofenadine & 2.8 & 6 & 501.65 & $30 \pm 7$ \\
\hline Ganciclovir & 0.7 & 99 & 255.20 & $9 \pm 7$ \\
\hline Gatifloxacin & 20 & 1 & 375.39 & $100 \pm 7$ \\
\hline Levocarnitine & 1.3 & 98 & 161.20 & $10 \pm 7$ \\
\hline Lisinopril & 0.6 & 45 & 405.49 & $26 \pm 7$ \\
\hline Lomefloxacin & 15 & 2 & 351.35 & $97 \pm 7$ \\
\hline Meloxicam & 13 & 1 & 351.40 & $97 \pm 7$ \\
\hline Melphalan & 84 & 0 & 305.20 & $100 \pm 12$ \\
\hline Mesalamine & 0.9 & 90 & 153.14 & $34 \pm 7$ \\
\hline Minocycline & 50 & 0 & 457.48 & $100 \pm 7$ \\
\hline Norfloxacin & 1.0 & 56 & 319.33 & $35 \pm 7$ \\
\hline Ofloxacin & 4.7 & 8 & 361.37 & $100 \pm 7$ \\
\hline Pefloxacin & 23 & 1 & 333.36 & $100 \pm 8$ \\
\hline Piroxicam & 20 & 1 & 331.35 & $100 \pm 7$ \\
\hline Sparfloxacin & 42 & 0 & 392.40 & $100 \pm 7$ \\
\hline Sulfadiazine & 1.2 & 45 & 246.20 & $100 \pm 7$ \\
\hline Terbutaline & 1.4 & 99 & 225.28 & $16 \pm 7$ \\
\hline Tetracycline & 3.8 & 6 & 444.43 & $83 \pm 7$ \\
\hline Torsemide & 22 & 1 & 348.42 & $82 \pm 7$ \\
\hline Trovafloxacin & 57 & 0 & 416.35 & $97 \pm 15$ \\
\hline
\end{tabular}

a Average absolute bioavailability taken from several sources [68, 70-71, 73-81] and corrected for hepatic clearance $[63,72]$ to generate the $F_{\text {a }}$ data. Errors are determined as a range of absolute bioavailability values spread over different literature sources. For those molecules where errors in the published $F$ values are not available, an average error value of $7 \%$ was used. 
This high paracellular fraction is somewhat surprising, given the discussions surrounding the human jejunal permeability measurements by Lennernäs and coworkers, who have suggested that the paracellular route may not be as important as that of the passive transcellular route in intestinal absorption for the 42 compounds (mostly drugs) tested in humans so far [66]. However, it could be that most of the compounds whose jejunal permeability has been determined are not ampholytes such as those considered here, although some molecules are included in both studies.

Figure 3.15 shows the fraction absorbed of the 33 ampholytes as a function of the $\log P_{\mathrm{e}}^{\mathrm{INT}}$ values deduced at the gradient $\mathrm{pH} 6.5$, with the solid curve representing the best fit to Equation 3.40. In the previous study, the analysis was based on the absolute bioavailability data [39]. Despite most of the molecules exhibiting low hepatic clearance, the bioavailability data have been converted to the absorbed fraction in this study. Re-analysis of the absorbed fraction data revealed a consistent picture [39]. The best-fitted parameters were: $H=100, G=$ $0.29 \times 10^{6} \mathrm{~cm}^{-1} \mathrm{~s}$ and $S=1.0$. The dotted curves were derived in the same way as

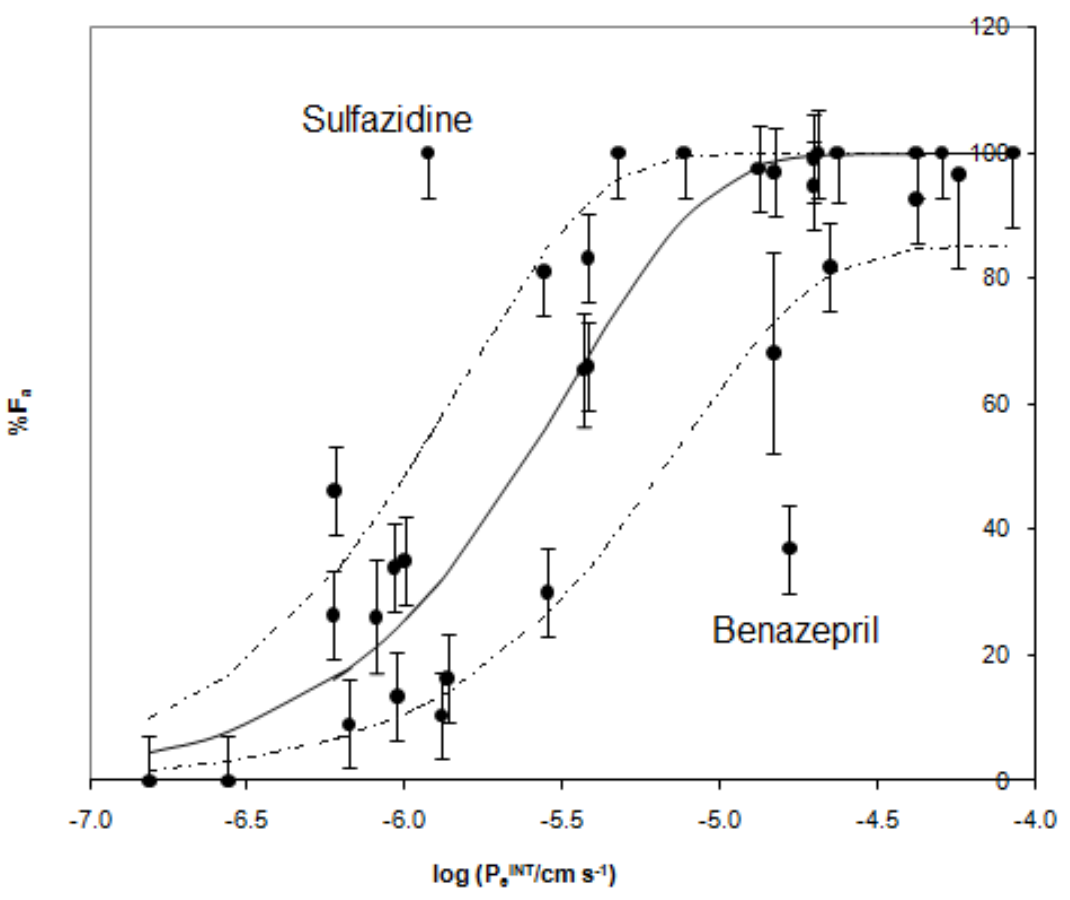

Figure 3.15. Correlation between fraction absorbed $\left(\% F_{a}\right)$ and the effective permeability (corrected for paracellular transport and unstirred water layer permeability (UWL thickness $=500 \mu \mathrm{m}$ )) for the 33 ampholytes studied.

The solid curve is the best fit of the function represented by Equation 3.40.

The dashed curves are based on estimated errors in the $\log P_{\mathrm{e}}{ }^{\text {INT }}$ values $(0.35)$. The outliers are labelled with the drug names 
in Figure 3.14. For most of the ampholytes studied in this work, the estimated errors in the $F$ data could be up to $\pm 16 \%$. This is based on the assumption of a $95 \%$ confidence interval from different published $\% F$ values, where available. In instances where errors in the published $\% F$ values were not available, an average value of $7 \%$ was assumed, based on known errors from the rest of the molecules. Based on this model, the $P_{\mathrm{e}}{ }^{\mathrm{INT}}$ values for the $50 \%$ predicted absorbed fraction in humans could be estimated to be $2.3 \times 10^{-6} \mathrm{~cm} \mathrm{~s}^{-1}$. It was noted that the values of $S$ were about 70 -fold greater than in the model using a rotating permeation cell (Section 3.4.3). This may be due to the different approaches used in calculating $P_{\mathrm{m}}$ values from the in vitro permeability data. As shown in Figure 3.15, the absorbed fraction data of most of the ampholytes was generally well-predicted by the approach described here. However, famotidine, sulfadiazine and ciprofloxacin were predicted as false negatives. This might have occurred due to the presence of facilitated transport mechanism(s) which the passive permeability model failed to account for. Benazepril was the only false positive. This molecule is an ester prodrug of benazeprilat. Following oral absorption, the prodrug is completely hydrolysed (primarily in the liver) to benazeprilat [67]. It is plausible that these data are under-estimated to some extent because of the fast hydrolysis reaction in vivo.

\subsection{CONCLUDING REMARKS}

PAMPA provides useful information to assess the passive permeability of research compounds across an artificial membrane. The method can be run with relatively low costs, compared to other cellular permeability assays. It was developed with the aim of enhanced throughput in mind, and can potentially be utilised in screening. Undoubtedly, this has been shown to be a success, evident by the fact that an increasing number of research laboratories are adopting PAMPA as part of their screening toolkit. In particular, PAMPA has an important role to play in supporting the development of chemical series and lead compounds in early drug discovery. For instance, in combination with other in vitro tests, PAMPA could form a part of a testing cascade in lead generation and/or lead optimisation campaigns. However, it is not sustainable to test every research compound using PAMPA. Instead, it would be more cost effective to apply this method in scenarios where passive permeability is likely to be an issue for the chemical series of interest.

The PAMPA method has improved considerably over the last decade. New lipid models have been developed to better mimic various cellular barriers, including intestinal epithelial layers and the blood-brain barrier. Pioneering PAMPA researchers soon realised the impact of unstirred water layers on the measured data, and subsequently developed the individual well stirring technique to control the thickness of the unstirred water layers. More recently, a novel rotating permeation cell, which offers precise control of the hydrodynamics in the donor and acceptor compartments, has been utilised to investigate the effects 
of unstirred water layers on the accuracy of PAMPA data. This has provided new insights into the initial rate of transport and loading of the molecules into the artificial membrane. While the rotating permeation cell can only study one compound at a time, there is the potential to further develop this technique into a miniaturised version, for example, which would offer throughput that is comparable to the current PAMPA-based microtitre plate.

Mathematical models have been developed to predict the paracellular transport of test compounds. PAMPA permeability data, corrected for paracellular transport, were generated on two sets of marketed drugs, one of which included amphoteric drugs predominately charged at physiological $\mathrm{pH}$. It was shown that these effective permeability data correlated well with data on the absorbed fraction in humans.

As the PAMPA technique evolves, it is expected that more practitioners will realise the existence and the effects of unstirred water layers, and carefully design their experiments to eliminate any artefacts that might be obtained from a static permeation study. To differentiate the change in permeation rates on analogues (with small structural changes) within a chemical series of interest will call for sensitive PAMPA data. It is envisaged that the research and optimisation of lipid models will continue to enhance the resolution of these data.

\section{Acknowledgements}

We gratefully acknowledge the inspiring discussion with Dr. Alex Avdeef on PAMPA methods. His advice on the paracellular transport model was invaluable. Thanks are especially due to Oksana Tsinman and Na Sun of pION Inc. for their contribution to the DS-PAMPA study on the amphoteric drugs described in this chapter.

\section{REFERENCES}

1. M.A. Navia, P.R. Chaturvedi. Design principles for orally bioavailable drugs. Drug Discovery Today 1(5) (1996) 179-189.

2. M.V.S. Varma, R.S. Obach, C. Rotter, H.R. Miller, G. Chang, S.J. Steyn, A. El-Kattan, M.D. Troutman. Physicochemical space for optimum oral bioavailability: contribution of human intestinal absorption and first-pass elimination. Journal of Medicinal Chemistry 53(3) (2010) 1098-1108.

3. P.D. Dobson, D.B. Kell. Carrier-mediated cellular uptake of pharmaceutical drugs: an exception or the rule? Nature Reviews Drug Discovery 7(3) (2008) 205-220

4. K. Sugano, M. Kansy, P. Artursson, A. Avdeef, S. Bendels, L. Di, G.F. Ecker, B. Faller, H. Fischer, G. Gerebtzoff, H. Lennernäs, F. Senner. Coexistence of passive and carrier-mediated processes in drug transport. Nature Reviews Drug Discovery 9(8) (2010) 597-614.

5. G.L. Amidon, H. Lennernäs, V.P. Shah, J.R. Crison. A theoretical basis for a biopharmaceutic drug classification: The correlation of in vitro drug product dissolution and in vivo bioavailability. Pharmaceutical Research 12(3) (1995) 413-420. 
6. Center for Drug Evaluation and Research. Food and Drug Administration. Guidance for Industry: Waiver of In Vivo Bioavailability and Bioequivalence Studies for Immediate-Release Solid Oral Dosage Forms Based on a Biopharmaceutics Classification System 2000. http://www.fda.gov/downloads/Drugs/GuidanceComplianceRegulatoryInformation/Guidances/UCM070246.pdf, $\quad$ accessed $\quad 12$ August 2011.

7. M. Kansy, F. Senner, K. Gubernator. Physicochemical high throughput screening: Parallel artificial membrane permeation assay in the description of passive absorption processes. Journal of Medicinal Chemistry 41(7) (1998) 1007-1010.

8. P. Artursson, J. Karlsson. Correlation between oral drug absorption in humans and apparent drug permeability coefficients in human intestinal epithelial (Caco-2) cells. Biochemical and Biophysical Research Communications 175(3) (1991) 880885.

9. V. Fade, S. Stavchansky. Link between drug absorption solubility and permeability measurements in Caco-2 cells. Journal of Pharmaceutical Sciences 87(12) (1998) 1604-1607.

10. J.D. Irvine, L. Takahashi, K. Lockhart, J. Cheong, J.W. Tolan, H.E. Selick,, J.R. Grove. MDCK (Madin-Darby canine kidney) cells: A tool for membrane permeability screening. Journal of Pharmaceutical Sciences 88(1) (1999) 28-33.

11. M.H. Jacobs. Some aspects of cell permeability to weak electrolytes. Cold Spring Harbor Symposia on Quantitative Biology 8 (1940) 30-39.

12. P.A. Shore, B.B. Brodie, C.A. Hogben. The gastric secretion of drugs: a pH partition hypothesis. The Journal of Pharmacology and Experimental Therapeutics 119(3) (1957) 361-369.

13. C.A. Hogben, D.J. Tocco, B.B. Brodie, L.S. Schanker. On the mechanism of intestinal absorption of drugs. The Journal of Pharmacology and Experimental Therapeutics 125(4) (1959) 275-282.

14. E.R. Garrett, P.B. Chemburkar. Evaluation, control, and prediction of drug diffusion through polymeric membranes. II. Diffusion of aminophenones through silastic membranes: a test of the pH-partition hypothesis. Journal of Pharmaceutical Sciences 57(6) (1968) 949-959.

15. V.F. Smolen. Misconceptions and thermodynamic untenability of deviations from pH-partition hypothesis. Journal of Pharmaceutical Sciences 62(1) (1973) 77-79.

16. A. Avdeef, K.J. Box, J.E.A. Comer, C. Hibbert, K.Y. Tam. pH-Metric $\log P 10$. Determination of liposomal membrane-water partition coefficients of ionizable drugs. Pharmaceutical Research 15(2) (1998) 209-215.

17. K. Iseki, T. Hirano, Y. Fukushi, Y. Kitamura, S. Miyazaki, M. Takada, M. Sugawara, H. Saitoh, K. Miyazaki. The $\mathrm{pH}$ dependent uptake of enoxacin by rat intestinal brushborder membrane vesicles. Journal of Pharmacy and Pharmacology 44(9) (1992) 722-726.

18. C. Ottiger, H. Wunderli-Allenspach. Partition behaviour of acids and bases in a phosphatidylcholine liposome-buffer equilibrium dialysis system. European Journal of Pharmaceutical Sciences 5(4) (1997) 223-231.

19. A. Adson, T.J. Raub, P.S. Burton, C.L. Barsuhn, A.R. Hilgers, N.F.H. Ho, K.L. Audus. Quantitative approaches to delineate paracellular diffusion in cultured epithelial cell monolayers. Journal of Pharmaceutical Sciences 83(11) (1994) 1529-1536. 
20. K. Palm, K. Luthman, J. Ros, J. Gråsjö, P. Artursson. Effect of molecular charge on intestinal epithelial drug transport: $\mathrm{pH}$ - dependent transport of cationic drugs. Journal of Pharmacology and Experimental Therapeutics 291(2) (1999) 435-443.

21. H. Lennernäs. Human jejuna effective permeability and its correlation with preclinical drug absorption models. Journal of Pharmacy and Pharmacology 49(7) (1997) 627-638.

22. H. Lennernäs. Animal data: The contributions of the Ussing chamber and perfusion systems to predicting human oral drug delivery in vivo. Advanced Drug Delivery Reviews 59(11) (2007) 1103-1120.

23. A. Avdeef. Absorption and Drug Development. Solubility, Permeability, and Charge State, Wiley-Interscience, 2003.

24. A. Avdeef, M. Strafford, E. Block, M. P. Balogh, W. Chambliss, I. Khan. Drug absorption in vitro model: filter-immobilized artificial membranes 2 . Studies of the permeability properties of lactones in Piper methysticum Forst. European Journal of Pharmaceutical Sciences 14(4) (2001) 271-280.

25. M. Velický, D.F. Bradley, K.Y. Tam, R.A.W. Dryfe. In situ artificial membrane permeation assay under hydrodynamic control: permeability-pH profiles of warfarin and verapamil. Pharmaceutical Research 27(8) (2010) 1644-1658.

26. J.C. Dearden, G.M. Bresnen. The measurement of partition coefficients. Quantitative Structure-Activity Relationships 7(3) (1988) 133-144.

27. A. Avdeef, P.E. Nielsen, O. Tsinman. PAMPA - A drug absorption in vitro model: 11. Matching the in vivo unstirred water layer thickness by individual-well stirring in microtitre plates. European Journal of Pharmaceutical Sciences 22(5) (2004) 365374.

28. A. Adson, P.S. Burton, T.J. Raub, C.L. Barsuhn, K.L. Audus, N.F.H. Ho. Passive diffusion of weak organic electrolytes across Caco-2 cell monolayers: Uncoupling the contributions of hydrodynamic, transcellular, and paracellular barriers. Journal of Pharmaceutical Sciences 84(10) (1995) 1197-1204.

29. V.G. Levich. Physicochemical Hydrodynamics. Englewood Cliffs, London, 1962.

30. J. Karlsson, P. Artursson. A method for the determination of cellular permeability coefficients and aqueous boundary layer thickness in monolayers of intestinal epithelial (Caco-2) cells grown in permeable filter chambers. International Journal of Pharmaceutics 71(1) (1991) 55-64.

31. G.E. Amidon, W.I. Higuchi, N.F.H. Ho. Theoretical and experimental studies of transport of micelle-solubilized solutes. Journal of Pharmaceutical Sciences 71(1) (1982) 77-84.

32. W.J. Albery, J.F. Burke, E.B. Leffler, J. Hadgraft. Interfacial Transfer Studied with a Rotating Diffusion Cell. Journal of the Chemical Society-Faraday Transactions I 72 (1976) 1618-1626.

33. R.H. Guy, D.H. Honda. Solute transport resistance at the octanol - water interface. International Journal of Pharmaceutics 19(2) (1994) 129-137.

34. D.E. Leahy, A.R. Wait. Solute transport resistance at water-oil interfaces. Journal of Pharmaceutical Sciences 75(12) (1986) 1157-1161.

35. F. Wohnsland, B. Faller. High-throughput permeability $\mathrm{pH}$ profile and highthroughput alkane/water log $\mathrm{P}$ with artificial membranes. Journal of Medicinal Chemistry 44(6) (2001) 923-930. 
36. M. Bermejo, A. Avdeef, A. Ruiz, R. Nalda, J.A. Ruell, O. Tsinman, I. Gonzalez, C. Fernandez, G. Sanchez, T.M. Garrigues, V. Merino. PAMPA - a drug absorption in vitro model 7. Comparing rat in situ, Caco-2, and PAMPA permeability of fluoroquinolones. European Journal of Pharmaceutical Sciences 21(4) (2004) 429441.

37. A. Avdeef. High-throughput Measurement of Permeability Profiles. Drug Bioavailability, Wiley-VCH Weinheim, 2003.

38. C. Dagenais, A. Avdeef, O. Tsinman, A. Dudley, R. Beliveau. P-glycoprotein deficient mouse in situ blood-brain barrier permeability and its prediction using an in combo PAMPA model. European Journal of Pharmaceutical Sciences 38(2) (2009) 121-137.

39. K.Y. Tam, A. Avdeef, O. Tsinman, N. Sun. The permeation of amphoteric drugs through artificial membranes - an in combo absorption model based on paracellular and transmembrane permeability. Journal of Medicinal Chemistry 53(1) (2010) 392-401.

40. K. Sugano, H. Hamada, M. Machida, H. Ushio, K. Saitoh, K. Terada. Optimized conditions of bio-mimetic artificial membrane permeation assay. International Journal of Pharmaceutics 228(1) (2001) 181-188.

41. K. Sugano, N. Takata, M. Machida, K. Saitoh, K. Terada. Prediction of passive intestinal absorption using bio-mimetic artificial membrane permeation assay and the paracellular pathway model. International Journal of Pharmaceutics 241(2) (2002) 241-251.

42. E.M. Renkin. Filtration, diffusion, and molecular sieving through porous cellulose membranes. The Journal of General Physiology 38(2) (1954) 225-243.

43. X. Chen, A. Murawski, K. Patel, C.L. Crespi, P.V. Balimane. A novel design of artificial membrane for improving the PAMPA model. Pharmaceutical Research 25(7) (2008) 1511-1520.

44. A. Avdeef, 0. Tsinman, K. Tsinman. Very thin PAMPA membranes indicate higher antipyrine permeability but are fragile and contain leaky water pores. AAPS meeting 2010. Poster presentation.

45. G.E. Flaten, A.B. Dhanikulaa, K. Luthman, M. Brandl. Drug permeability across a phospholipid vesicle based barrier: A novel approach for studying passive diffusion. European Journal of Pharmaceutical Sciences 27(1) (2006) 80-90.

46. G. Corti, F. Maestrelli, M. Cirri, S. Furlanetto, P. Mura. Development and evaluation of an in vitro method for prediction of human drug absorption I. Assessment of artificial membrane composition. European Journal of Pharmaceutical Sciences 27(4) (2006) 346-353.

47. G. Corti, F. Maestrelli, M. Cirri, N. Zerroukb, P. Mura. Development and evaluation of an in vitro method for prediction of human drug absorption II. Demonstration of the method suitability. European Journal of Pharmaceutical Sciences 27(4) (2006) 354-362.

48. Z.S. Teksin, P.R. Seo, J.E. Polli. Comparison of drug permeabilities and BCS classification: Three lipid-component PAMPA system method versus caco-2 monolayers. The American Association of Pharmaceutical Scientists Journal 12 (2006) 238-241. 
49. L. Di, E.H. Kerns, K. Fan, O.J. McConnell, G.T. Carter. High throughput artificial membrane permeability assay for blood-brain barrier. European Journal of Medicinal Chemistry 38(3) (2003) 223-232.

50. A. Avdeef, 0. Tsinman. PAMPA - A drug absorption in vitro model: 13. Chemical selectivity due to membrane hydrogen bonding: In combo comparisons of HDM-, DOPC-, and DS-PAMPA models. European Journal of Pharmaceutical Sciences 28(1) (2006) 43-50.

51. H. Lennernäs. Human intestinal permeability. Journal of Pharmaceutical Sciences 87(4) (1998) 403-410.

52. A.A. Cools, L.H.M. Janssen. Influence of sodium ion-pair formation on transport kinetics of warfarin through octanol-impregnated membranes. Journal of Pharmacy and Pharmacology 35(10) (1983) 689-691.

53. A. Walter, J. Gutknecht. Monocarboxylic acid permeation through lipid bilayer membranes. Journal of Membrane Biology 77(3) (1984) 255-264.

54. J.B. Dressman, G.L. Amidon, C. Reppas, V.P. Shah. Dissolution testing as a prognostic tool for oral drug absorption: Immediate release dosage forms. Pharmaceutical Research 15(1) (1998) 11-22.

55. K. Kontturi, L. Murtomäki, J.A. Manzanares. Ionic Transport Processes in Electrochemistry and Membrane Science. Oxford University Press, 2008.

56. G.L. Flynn, S.H. Yalkowsky, T.J. Roseman. Mass transport phenomena and models: theoretical concepts. Journal of Pharmaceutical Sciences 63(4) (1974) 479-510.

57. G.L. Amidon, P.J. Sinko, D. Fleisher. Estimating human oral fraction dose absorbed: A correlation using rat intestinal membrane permeability for passive and carriermediated compounds. Pharmaceutical Research 5(10) (1988) 651-654.

58. A. Avdeef. Leakiness and size exclusion of paracellular channels in cultured epithelial cell monolayers-interlaboratory comparison. Pharmaceutical Research 27(3) (2010) 480-489.

59. A. Avdeef. The rise of PAMPA. Expert Opinion on Drug Metabolism \& Toxicology 1(2) (2005) 325-342.

60. A. Avdeef, P. Artursson, S. Neuhoff, L. Lazorova, J. Gråsjö, S. Tavelin. Caco-2 permeability of weakly basic drugs predicted with the double-sink PAMPA pKa flux method. European Journal of Pharmaceutical Sciences 24(4) (2005) 333-349.

61. D. Winne. Dependence of intestinal absorption in vivo on the unstirred layer. Naunyn-Schmiedeberg's Archives of Pharmacology 304 (1978) 175-181.

62. U. Fagerholm, H. Lennernäs. Experimental estimation of the effective unstirred water layer thickness in the human jejunum, and its importance in oral drug absorption. European Journal of Pharmaceutical Sciences 3(5) (1995) 247-253.

63. R.S. Obach, F. Lombardo, N.J. Waters. Trend analysis of a database of intravenous pharmacokinetic parameters in human for 670 drug compounds. Drug Metabolism and Disposition 36(7) (2008) 1385-1405.

64. L.X. Yu, G.L. Amidon. A compartmental absorption and transit model for estimating oral drug absorption. International Journal of Pharmaceutics 186(2) (1999) 119125.

65. M. Velický, K.Y. Tam, R.A.W. Dryfe. In situ artificial membrane permeation assay under hydrodynamic control: Correlation between drug in vitro permeability and 
fraction absorbed in humans. European Journal of Pharmaceutical Sciences 44(3) (2011) 299-309.

66. H. Lennernäs. Intestinal permeability and its relevance for absorption and elimination. Xenobiotica 37(10) (2007) 1015-1051.

67. G. Kaiser, R. Ackermann, S. Brechbühler, W. Dieterle. Pharmacokinetics of the angiotensin converting enzyme inhibitor benazepril.HCl (CGS 14824 A) in healthy volunteers after single and repeated administration. Biopharmaceutics and Drug Disposition 10(4) (1989) 365-376.

68. W.K. Sietsema. The absolute oral bioavailability of selected drugs. International Journal of Clinical Pharmacology Therapy and Toxicology 27(10) (1989) 179-211.

69. D. Tenero, D. Martin, B. Ilson, J. Jushchyshyn, S. Boike, D. Lundberg, N. Zariffa, D. Boyle, D. Jorkasky. Pharmacokinetics of intravenously and orally administered eprosartan in healthy males: Absolute bioavailability and effect of food. Biopharmaceutics and Drug Disposition 19(6) (1998) 351-356.

70. C.T. Dollery. Therapeutic Drugs. Churchill Livingstone, 1999.

71. FDA Drug Information (http://www.fda.gov/Drugs/default.htm).

72. L.S. Goodman, A.G. Gilman, J.G. Hardman, L.E. Limbird. Goodman \& Gilman's the pharmacological basis of therapeutics. McGraw-Hill, 2006.

73. G. Lappin, Y. Shishikura, R. Jochemsen, R.J. Weaver, C. Gesson, B. Houston, B. Oosterhuis, O.J. Bjerrum, M. Rowland, C. Garner. Pharmacokinetics of fexofenadine: Evaluation of a microdose and assessment of absolute oral bioavailability. European Journal of Pharmaceutical Sciences 40(2) (2010) 125-131.

74. F.P. LaCreta, S. Kaul, G.D. Kollia, G. Duncan, D.M. Randall, D.M. Grasela. Interchangeability of 400-mg intravenous and oral gatifloxacin in healthy adults. Pharmacotherapy 20(6) (2000) 59S-66S.

75. C. Chen. Physicochemical, pharmacological and pharmacokinetic properties of the Zwitterionic antihistamines cetirizine and levocetirizine. Current Medicinal Chemistry 15(21) (2008) 2173-2191.

76. R. Whomsley, M.S. Benedetti. Development of new H1 antihistamines: the importance of pharmacokinetics in the evaluation of safe and therapeutically effective agents. Current Medicinal Chemistry - Anti-Inflammatory \& Anti-Allergy Agents 4(5) (2005) 451-464.

77. K.N. Agwuh, A. MacGowan. A. Pharmacokinetics and pharmcodynamics of the tetracyclines including glycylcyclines. Journal of Antimicrobial Chemotherapy 58(2) (2006) 256-265.

78. P.H. Hinderling. Evaluation of a novel method to estimate absolute bioavailability of drugs from oral data. Biopharmaceutics \& Drug Disposition 24(1) (2003) 1-16

79. Physician's Desk Reference, PDR Network LLC, 2009.

80. A.M. Frydman, Y. Leroux, M.A. Lefebvre, F. Djebbar, J.B. Fourtillan, J. Gaillot. Pharmacokinetics of pefloxacin after repeated intravenous and oral-administration (400-Mg Bid) in young healthy-volunteers. Journal of Antimicrobial Chemotherapy 17 Suppl B (1986) 65-79.

81. R. Teng, L.C. Dogolo, S.A. Willavize, H.L. Friedman, J. Vincent. Oral bioavailability of trovafloxacin with and without food in healthy volunteers. Journal of Antimicrobial Chemotherapy 39 Suppl B (1997) 87-92. 